\title{
Dynamics of the Water Molecules at the Intrinsic Liquid Surface as Seen from Molecular Dynamics Simulation and ITIM Analysis
}

Balázs Fábián, Milan V. Sen\#anski, Ilija N. Cvijeti\#, Pal Jedlovszky, and George Horvai

J. Phys. Chem. C, Just Accepted Manuscript • DOI: 10.1021/acs.jpcc.5b10370 • Publication Date (Web): 04 Apr 2016

Downloaded from http://pubs.acs.org on April 10, 2016

\section{Just Accepted}

"Just Accepted" manuscripts have been peer-reviewed and accepted for publication. They are posted online prior to technical editing, formatting for publication and author proofing. The American Chemical Society provides "Just Accepted" as a free service to the research community to expedite the dissemination of scientific material as soon as possible after acceptance. "Just Accepted" manuscripts appear in full in PDF format accompanied by an HTML abstract. "Just Accepted" manuscripts have been fully peer reviewed, but should not be considered the official version of record. They are accessible to all readers and citable by the Digital Object Identifier (DOI®). "Just Accepted" is an optional service offered to authors. Therefore, the "Just Accepted" Web site may not include all articles that will be published in the journal. After a manuscript is technically edited and formatted, it will be removed from the "Just Accepted" Web site and published as an ASAP article. Note that technical editing may introduce minor changes to the manuscript text and/or graphics which could affect content, and all legal disclaimers and ethical guidelines that apply to the journal pertain. ACS cannot be held responsible for errors or consequences arising from the use of information contained in these "Just Accepted" manuscripts. 


\title{
Dynamics of the Water Molecules at the Intrinsic Liquid
} Surface as Seen from Molecular Dynamics Simulation and ITIM Analysis

\author{
Balázs Fábián, ${ }^{1,2}$ Milan V. Senćanski, ${ }^{3}$ Ilija N. Cvijetić, ${ }^{4}$ Pál \\ Jedlovszky, ${ }^{5,6,7, *}$ and George Horvai, ${ }^{1,5, *}$ \\ ${ }^{1}$ Department of Inorganic and Analytical Chemistry, Budapest University of \\ Technology and Economics, Szt. Gellért tér 4, H-1111 Budapest, Hungary \\ ${ }^{2}$ Institut UTINAM-UMR CNRS 6213, Faculté des Sciences, Université de \\ Franche-Comté, F-25030 Besançon Cedex, France \\ ${ }^{3}$ Center for Multidisciplinary Research Institute of Nuclear Sciences Vinča, \\ P.O.Box 522, RS-11001 Belgrade, Serbia \\ ${ }^{4}$ Innovation Center, Faculty of Chemistry, University of Belgrade, Studentski trg \\ 12-16, Belgrade, Serbia \\ ${ }^{5}$ MTA-BME Research Group of Technical Analytical Chemistry, Szt. Gellért tér \\ 4, H-1111 Budapest, Hungary \\ ${ }^{6}$ EKF Department of Chemistry, Leányka utca 6, H-3300 Eger, Hungary \\ ${ }^{7}$ Laboratory of Interfaces and Nanosize Systems, Institute of Chemistry, Eötvös \\ Loránd University, Pázmány P. Stny 1/A, H-1117 Budapest, Hungary
}

Running title: Dynamics of Water at the Intrinsic Liquid Surface

*Electronic mail: pali@chem.elte.hu (PJ), george.horvai@mail.bme.hu (GH) 


\begin{abstract}
$\underline{\text { Abstract }}$
Dynamical properties at the liquid-vapor interface of water are investigated at $298 \mathrm{~K}$ on the basis of molecular dynamics simulations and intrinsic surface analysis. Mean surface residence time and diffusion coefficient of the molecules as well as $\mathrm{H}$-bond lifetimes are calculated at the liquid surface, and compared to the bulk values. It is found that surface molecules have a non-negligible diffusion component along the surface normal, although this component is limited in time to 7-15 ps, a value comparable with the mean surface residence time. It is also seen that interfacial molecules move considerably faster, and their H-bonds live shorter than in the bulk liquid phase. This finding is explained by the relation between the number of H-bonded neighbors and mobility, namely that molecules being tethered by more H-bonds move slower, and their H-bonds live longer than in the case of molecules of less extensive H-bonding. Finally, it is found that molecules residing long at the surface are clustering around each other, forming more and longer living $\mathrm{H}$-bonds within the surface layer, but much less outside this layer than other interfacial molecules, indicating that longer surface residence is related to weaker interaction with the subsurface region.
\end{abstract}




\section{Introduction}

Molecular level investigation of the properties of soft or fluid interfaces (e.g., the liquid-liquid and liquid-vapor interface) became in the focus of intensive scientific investigations in the past two decades. Such soft interfaces play a key role in a number of physical, chemical and biological processes and in their industrial applications, from heterogeneous catalysis to chromatography, from the local increase of concentrations (adsorption) to charge transfer, or from the metabolism of living cells to extraction. While the continuously increasing scientific interest in such systems is motivated by the important role soft interfaces play in various areas of science and technology, investigation of such systems has been enabled by the recent development of various surface sensitive experimental techniques, such as nonlinear spectroscopic methods ${ }^{1}$ (e.g., vibrational sum frequency generation $^{2,3}$ and second harmonic generation ${ }^{4-6}$ spectroscopies), reflection techniques, ${ }^{7}$ such as ${\mathrm{x}-\text { ray }^{8}}^{8}$ and neutron ${ }^{9}$ reflection, or time dependent fluorescence anisotropy ${ }^{10,11}$ measurements. Such experimental methods can be well complemented by computer simulations, ${ }^{12}$ the application of which to various soft interfaces has also been enabled in the past decades, by the rapid development of routinely available computing capacities.

Meaningful investigation of interfaces by computer simulations evidently requires that the molecules located at the surface of their phase have to be unambiguously identified. It is easy to show that this task is equivalent with finding the exact location of the surface at every point along its macroscopic plane. In the case of soft interfaces this is not an easy task at all, because such interfaces are corrugated, at the molecular length scale, by capillary waves. However, in the vast majority of the early interfacial simulations the problem caused by the capillary waves was simply neglected, and instead of the real, capillary wave corrugated, intrinsic interface a planar slab parallel with a face of the basic simulation box was considered. It was shown later several times that this neglect of the capillary waves and nonintrinsic treatment of the interface leads to systematic error of unknown magnitude concerning not only the structure ${ }^{13,14}$ or composition ${ }^{15-17}$ of the interfacial layer, but it can even propagate to the thermodynamic properties of the system. ${ }^{18}$

In spite of this regretful practice of neglecting the effect of the capillary waves, the need for a physically sound definition of the interface was already realized in the very first fluid interface simulations, in the pioneering works of Linse ${ }^{19}$ and Benjamin. ${ }^{20-22}$ In their studies they divided the system into slabs along the interface normal axis, and detected the 
position of the interface in each slab separately. Almost two decades later, Jorge and Cordeiro determined the number of slabs required to obtain convergent results. ${ }^{23,24}$ Berkowitz et al. used a Voronoi tessellation in the macroscopic plane of the interface, and lifted the Voronoi cells to the positions of their central particles along the macroscopic surface normal. ${ }^{25}$ In their pioneering work Chacón and Tarazona described a method to find the surface of minimum area that covers a set of pivot atoms, the list of which is determined in a self-consistent iterative process. ${ }^{26}$ Chowdhary and Ladanyi used a criterion based on the distance from the opposite phase molecules to determine the list of the interfacial molecules at the liquid-liquid interface. ${ }^{27}$ It was later shown by Jorge et al. that if a scalable parameter is introduced, this algorithm also gives comparable results with other intrinsic analysis methods. ${ }^{28}$ Further, several methods that are even free from the assumption that the interface is macroscopically planar have also been proposed in the past years. ${ }^{29-31}$ Previously we proposed another algorithm, called Identification of the Truly Interfacial Molecules (ITIM) to detect the surface molecules at soft interfaces. ${ }^{13}$ The ITIM method was shown to provide comparable results with alternative intrinsic methods, ${ }^{28,31}$ and was found to represent an excellent compromise between computational cost and accuracy. ${ }^{28}$

Once the real, intrinsic interface is located, various properties related to this interface can be calculated. Such properties include the profiles of various thermodynamic quantities (e.g., density, ${ }^{26,32}$ energy, ${ }^{33}$ pressure, ${ }^{33}$ solvation free energy, ${ }^{34}$ surface tension, ${ }^{33}$ electrostatic potential, ${ }^{35}$ etc.) relative to the position of the intrinsic interface. Such intrinsic profiles, determined either as continuous functions or their values layer-by-layer, ${ }^{33}$ proved to be essential in interpreting a number of surface-related phenomena, such as explaining the surface tension anomaly of water ${ }^{36,37}$ or the adhesive properties of Newton black films, ${ }^{38}$ addressing the plausibility of the 'HCN World' hypothesis, ${ }^{17}$ describing the surface properties of ionic liquids, ${ }^{39-42}$ aqueous electrolyte solution ${ }^{35}$ and water-oil interfaces, ${ }^{14,18,43}$ or analyzing the immersion depth of surfactants in water. ${ }^{44}$

In our previous paper we started a systematic investigation of various surface-related properties at liquid-vapor interfaces. ${ }^{33}$ We demonstrated the applicability of intrinsic analysis methods in describing surface thermodynamic properties. ${ }^{33}$ In the present paper we continue this systematic investigation and focus our attention to the analysis of the dynamic properties of the molecules at the liquid surface. In spite of the large number of simulation studies concerning dynamic properties of the molecules at fluid interfaces, ${ }^{45-50}$ (for a recent review, see Ref. 51) we are only aware of two such studies in which the intrinsic liquid surface was considered. Thus, Chowdhary and Ladanyi studied the dynamics of water molecules at 
various water/hydrocarbon liquid-liquid interfaces. ${ }^{52}$ However, in this study they still used a semi-intrinsic approach, namely they divided the surface region to several zones according to the behavior of the density profiles rather than considering the subsequent molecular layers beneath the surface. Further, the method they used to detect the intrinsic liquid surface was later shown to miss a large number of truly interfacial molecules, ${ }^{27}$ unless a scalable parameter is introduced in the analysis. ${ }^{28}$ A detailed study concerning the dynamics of the particles at the intrinsic liquid surface was also performed by Duque et al., considering the liquid-vapor interface of Lennard-Jones systems. ${ }^{53}$ Here we have chosen the liquid-vapor interface of water, in order to address also the role of hydrogen bonds in determining the dynamical properties at the liquid surface. The surface of liquid water has been studied extensively both by experimental and computer simulation methods in the past decade. The main findings of these studies are that the interfacial region, i.e., the layer within which the properties of water differs considerably from those in the bulk phase is very narrow, extending to only one or two molecular layers, ${ }^{13,54-58}$ the molecular structure of the water surface is highly mobile and dynamic, ${ }^{56}$ the strength of the hydrogen bonds at the surface does not differ much from that in the bulk phase, ${ }^{54,55,58}$ a large number of the surface $\mathrm{OH}$ groups are dangling towards the vapor phase, ${ }^{59-61}$ and that excitations of the $\mathrm{OH}$ stretch vibrational excitations are delocalized over several molecules. ${ }^{62-64}$ Here we focus our attention to the questions (i) whether the diffusion of the molecules is different at the liquid surface from that in the bulk liquid phase, and if so, how; (ii) whether the fact that certain molecules stay long at the liquid surface is related to some other properties (e.g., diffusion, hydrogen bonding, spatial correlation) of these molecules or it is a purely random choice; and (iii) how much are the lifetime and strength of the hydrogen bonds at the liquid surface different from those in the bulk liquid phase.

The paper is organized as follows. Details of the computer simulations and ITIM analyses performed are given in sec. 2 . The obtained results concerning the above questions are presented and discussed in detail in sec. 3. Finally, in sec. 4 the main conclusions of this study are summarized.

\section{Computational Details}

2.1. Molecular Dynamics Simulations. Molecular dynamics simulations of the liquid-vapor interface of water has been performed on the canonical $(N, V, T)$ ensemble at the 
temperature of $298 \mathrm{~K}$ with 4000 water molecules. For reference, bulk liquid water has also been simulated under identical thermodynamic conditions. To demonstrate that the obtained results are model independent, the simulations have been repeated with three different water models, namely the three-site $\mathrm{SPC} / \mathrm{E},{ }^{65}$ the four-site TIP4P, ${ }^{66}$ and the five site TIP5P-E ${ }^{67}$ ones. The $Y Z$ edges of the basic simulation box, being parallel with the macroscopic plane of the liquid surface, have been $50 \AA$ long, whereas the length of the macroscopic surface normal axis, $X$, has been $200 \AA$. Standard periodic boundary conditions have been applied.

The simulations have been performed using the GROMACS 4.5 .5 program package. ${ }^{68}$ Equations of motion have been integrated in time steps of $1 \mathrm{fs}$. All potential models considered have been rigid; the geometry of the water molecules has been kept unchanged by means of the LINCS algorithm. ${ }^{69}$ The temperature of the systems has been controlled using the Nosé-Hoover thermostat with the time constant of 1 ps. ${ }^{70,71}$ All interactions have been truncated to zero beyond the oxygen-oxygen cut-off distance of $15 \AA$; the long range part of the electrostatic interaction has been taken into account using the Particle Mesh Ewald (PME) method in its smooth particle implementation. ${ }^{72}$

At the beginning of each simulation 4000 water molecules were placed into a rectangular basic simulation box, the $X, Y$, and $Z$ edges of which have been 49,50 and $50 \AA$, respectively. Following energy minimization and a short equilibration, the systems were equilibrated on the isothermal-isobaric $(N, p, T)$ ensemble at 1 bar for $2 \mathrm{~ns}$ in such a way that the $Y$ and $Z$ edges of the basic box were kept fixed, and only edge $X$ was allowed to change. Pressure was controlled by the Parrinello-Rahman barostat. ${ }^{73}$ The length of axis $X$ was then fixed at its equilibrium value at 1 bar, and the systems were further equilibrated, now on the $(N, V, T)$ ensemble, for another $2 \mathrm{~ns}$. The density of the bulk liquid systems simulated this way agreed within $0.5 \%$ with the experimental density value of $0.997 \mathrm{~g} / \mathrm{cm}^{3}$ at $298 \mathrm{~K}^{74}$ in each case. Following equilibration, 2000 sample configurations of bulk liquid water, separated by 1 ps long trajectory each, have been saved for reference calculations. Then the $X$ edge length of the basic box was increased to $200 \AA$, and thus two liquid-vapor interfaces were created in the basic box. The interfacial systems were equilibrated again for 2 ns, and then 2000 sample configurations per system, separated by 1 ps long trajectory each, have been saved in the $2 \mathrm{~ns}$ long production runs. Finally, another 1000 sample configurations of each bulk or interfacial system considered, separated by 0.1 ps long trajectories each, have also been dumped for the hydrogen bond lifetime analyses. 
2.2. ITIM Analysis. The intrinsic surface of the liquid phase has been determined using the ITIM method ${ }^{13}$ in all sample configurations resulted from the interfacial simulations. The ITIM method is based on the concept that any meaningful definition of the surface molecules should account for the fact that surface molecules are the ones that are accessible also from the opposite phase. Thus, in an ITIM analysis a probe sphere of a given radius is moved along test lines perpendicular to the macroscopic plane of the surface, starting from the bulk opposite phase. Once this probe touches the first molecule of the phase of interest, this molecule is marked as being interfacial (as it is "seen" by the probe from the opposite phase), and the probe starts moving along the next test line. Once all test lines are considered, the full list of the interfacial molecules is obtained. ${ }^{13}$ An additional advantage of the method is that by disregarding the already identified molecules and repeating the entire procedure the molecules that constitute the subsequent subsurface layers can also be identified. $^{13}$

In the present ITIM analyses a probe sphere of the radius of $1.25 \AA$ has been used, in accordance with the suggestion of Jorge et al. ${ }^{28}$ The test lines along which the probe sphere was moved have been arranged in a $100 \times 100$ grid, thus, the distance of two neighboring test lines has been $0.5 \AA$. The surface layer of the liquid phase, as determined by the ITIM method, is illustrated in Figure 1, showing an equilibrium snapshot of the SPC/E system. Since here we are interested in the dynamics of the surface water molecules only, no further molecular layers beneath the surface one have been determined. Finally, all properties calculated have been averaged not only over the dumped sample configurations, but also over the two liquid-vapor interfaces present in the basic box.

2.3. Determination of the Mean Surface Residence Time. In order to calculate the mean residence time of the molecules at the liquid surface, $\tau_{\text {surf }}$, the survival probability, $L(t)$, i.e., the probability that a water molecule that is at the surface at $t_{0}$ remains at the surface until $t_{0}+t$ needs to be calculated. Since the departure of a molecule from the liquid surface is a process of first order kinetics, the survival probability, $L(t)$, is a function of exponential decay. Thus, fitting the function $\exp \left(-t / \tau_{\text {surf }}\right)$ to the simulated $L(t)$ data can readily provide the mean

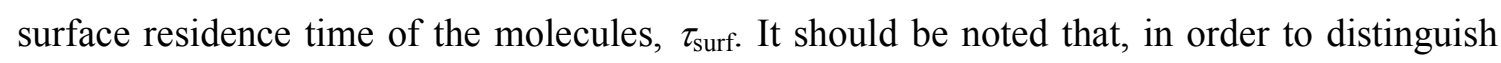
between the situations when a molecule leaves the surface temporarily, due to some fast oscillatory move, and when it leaves the surface permanently, departure of a molecule from the surface is conventionally allowed given that it returns to the surface layer within a short 
time window of $\Delta t$. For simplicity, here we use the $\Delta t$ value of $1 \mathrm{ps}$. Since $1 \mathrm{ps}$ is also the length of the trajectories between two consecutive sample configurations, this choice of $\Delta t$ practically means that no departure of the molecules from the surface layer is allowed; if a molecule is not found within the surface layer at any instant it is immediately regarded as having left the surface. To check whether this particular, simple choice of $\Delta t$ has any effect on the results, we have repeated all the analyses using $\Delta t=2 \mathrm{ps}$ (i.e., allowing a molecule to be absent from the surface layer in a snapshot, given that it is back in the next one), but this choice has left all of our conclusions unchanged. Therefore, all results presented here correspond to the simple choice of $\Delta t=1 \mathrm{ps}$, unless it is indicated otherwise.

\subsection{Calculation of the Diffusion Coefficient and the Characteristic Time of Diffusion.}

The diffusion coefficient of the molecules, $D$, can be calculated through the Einstein relation ${ }^{12}$ as

$$
D=\frac{M S D}{k t}
$$

where the value of $k$ is 2, 4, and 6 for one-, two-, and three-dimensional diffusion, respectively, and $M S D$ is the mean square displacement of the molecules within the time $t$ :

$$
M S D=\left\langle\left|\mathbf{r}_{i}\left(t_{0}+t\right)-\mathbf{r}_{i}\left(t_{0}\right)\right|^{2}\right\rangle
$$

In this equation the brackets $<\ldots>$ denote ensemble averaging, whereas $\mathbf{r}_{i}\left(t_{0}\right)$ and $\mathbf{r}_{i}\left(t_{0}+t\right)$ are the position vectors of particle $i$ at the times $t_{0}$ and $t_{0}+t$, respectively. In the case of three dimensional diffusion $\mathbf{r}_{i}$ is a spatial vector, while for two-dimensional diffusion it is a vector lying, by definition, within the plane in which diffusion is studied. The diffusion coefficient can thus be determined from the steepness of the straight line fitted to the simulated $M S D(t)$ data. It should be noted that this fitting should be performed up to a sufficiently low $t$ value to make sure that no particle diffuse more than half the simulation box edge length in any direction, and hence the MSD values are not yet affected by the periodic boundary conditions. In our case, the time range up to 35 ps probed to be sufficient for this purpose.

The characteristic time of a diffusive motion, $\tau_{\mathrm{D}}$, can be given as the time needed that a particle fully explore the surface area (in two dimensions) or volume (in three dimensions) it occupies (i.e., the surface area per molecule, $A_{\mathrm{m}}$, and volume per molecule, $V_{\mathrm{m}}$, respectively). More precisely, assuming that diffusion can be regarded as a random walk of the molecules, 
$\tau_{\mathrm{D}}$ is the time after which the positions visited by a molecule follow Gaussian distribution with the width of $\sqrt{A_{m}}$ (in two dimensions) and $\sqrt[3]{V_{m}}$ (in three dimensions). ${ }^{75}$ The value of $\tau_{\mathrm{D}}$ can thus be calculated in two dimensions as

$$
\tau_{\mathrm{D}}=\frac{A_{\mathrm{m}}}{4 D}
$$

where

$$
A_{\mathrm{m}}=\frac{2 Y Z}{N_{\text {surf }}},
$$

$N_{\text {surf }}$ being the number of the surface molecules in the system.

\section{Results and Discussion}

3.1. Density Profiles. The molecular number density profiles of the entire interfacial systems simulated as well as of the surface molecular layer of their liquid phase are shown in Figure 2. As is expected, the three water models considered resulted in very similar density profiles. The density peak of the surface layer extends well into the $X$ range where the density of the system already reaches its bulk liquid phase value, indicating that without using an intrinsic surface analysis method a large fraction of the truly interfacial molecules would have not been detected. Further, the density peak of the surface layer does not fully account for the intermediate density part of the overall density profile, indicating that without using an intrinsic surface analysis method a large number of non-interfacial water molecules would have been (erroneously) taken into account in the analyses. The density profile of the surface molecular layer is of Gaussian shape, in accordance with former theoretical considerations. ${ }^{76}$ The width of this Gaussian at half maximum, $d$ (see the inset of Fig. 2), will serve as a measure of the width of the surface layer in the following analyses.

3.2. Residence Time of the Molecules at the Surface. Before analyzing the dynamical properties of the water molecules at the liquid surface, we have to determine the time scale of their existence within the surface layer. The dynamics of the surface and bulk 
phase molecules can only be distinguished from each other in processes the time scale of which is considerably shorter than the typical time a molecule stays at the liquid surface.

The survival probability of the molecules within the surface layer, $L(t)$, is shown in Figure 3 as obtained with the three water models considered. To emphasize their exponential decay, the inset of Fig. 3 shows the simulated $L(t)$ data on a logarithmic scale. To avoid any disturbing effect of the fast, oscillatory moves of the molecules, the $\exp \left(-t / \tau_{\text {surf }}\right)$ function has only been fitted to the data points above 20 ps. The fitted functions are also shown in Fig. 3.

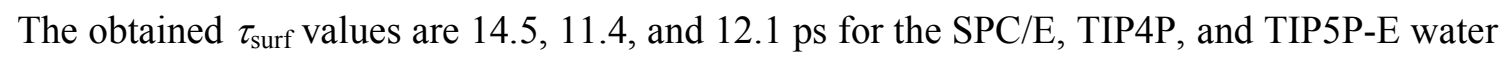
models, respectively. These values, collected in Table 1, set the time scale of processes that can be meaningfully analyzed within the surface layer below the order of about $10 \mathrm{ps}$.

3.3. Diffusion in the Surface Layer First we have calculated the three-dimensional diffusion coefficient of the surface molecules (their $M S D$ values being calculated solely in the period they belong to the surface layer). To check whether the speed of the diffusion at the surface is related to the surface residence time of the molecules in any way, we have selected those molecules of the surface layer that will reside at the surface for sufficiently long time. For this, we have used two different criteria: we have selected the longest residing $20 \%$ and $10 \%$ of the surface molecules in each frame, and calculated also the diffusion coefficients of solely these long-residing surface molecules. In doing this, first we determined the surface residence time corresponding to the $20 \%$ and $10 \%$ longest residing molecules from the $L(t)$ data (Fig. 3), and we selected those surface molecules in each snapshot that survive at least that long at the surface from this snapshot on. Further, since the intrinsic liquid surface is a wavy two-dimensional object, we have also calculated the two-dimensional diffusion coefficients of all the surface molecules as well as of the longest residing $20 \%$ and $10 \%$ of them within the macroscopic plane of the liquid surface, $Y Z$, as well as their one-dimensional diffusion coefficients along the macroscopic surface normal axis, $X$. Finally, for comparisons, we have also calculated the diffusion coefficient in bulk water, in three dimensions, in two dimensions within an arbitrarily chosen plane, and in one dimension along an arbitrarily chosen line. The MSD vs. $t$ data corresponding to the above one-, two- and three-dimensional diffusion coefficients are shown in Figure 4 as obtained in SPC/E water, whereas the obtained diffusion coefficient values are collected in Table 2. Similar results have been obtained with the other two water models considered (data not shown).

Before going to a detailed analysis of the diffusion of the surface molecules, it has to be verified that the characteristic time of the diffusion at the liquid surface is indeed well 
below the mean surface residence time of the molecules. The $\tau_{\mathrm{D}}$ values of the surface molecules calculated considering their two-dimensional diffusion are included and compared with $\tau_{\text {surf }}$ in Table 1. As is seen, the $\tau_{\mathrm{D}}$ values are at least 3.5-4 times smaller than $\tau_{\text {surf }}$ in every case, indicating that diffusion of the molecules at the liquid surface occurs on a reasonably shorter time scale than the exchange of the molecules between the liquid surface and the bulk liquid phase, and hence surface diffusion can indeed be meaningfully analyzed. This is also illustrated by Figure 5, showing the trajectory of three arbitrarily chosen surface molecules the surface residence time of which is close to the average value, and that of three longresiding ones within the macroscopic surface plane, $Y Z$, as taken out from the simulation of $\mathrm{SPC} / \mathrm{E}$ water. As is seen, even average-residing molecules explore a reasonable surface portion while they are at the liquid surface, whereas long-residing molecules can explore rather large portions of the liquid surface. It should be noted that this behavior is in a clear contrast with the finding of Duque et al., obtained for the surface of Lennard-Jones liquids. ${ }^{53}$ Namely, in such systems, the characteristic time of the two-dimensional diffusion of the particles within the surface layer turned out to be almost equal to the mean surface residence time at every temperature considered. ${ }^{53}$ This contrast between the results obtained for the water surface and that of a Lennard-Jones fluid suggests that the extensive hydrogen bonding between the molecules at the water surface ${ }^{36,37}$ has a strong influence on the surface dynamics of the molecules, presumably by substantially increasing the mean residence time of the molecules at the liquid surface. This point will be further investigated in this paper.

In analyzing the obtained results it is seen that there is no difference between the diffusion coefficient of all surface molecules and that of the longest residing ones in any case, indicating that the residence time of the individual molecules at the liquid surface is independent from their diffusive motion. When comparing the two- and three-dimensional diffusion coefficients of the different molecules it is seen that the ratio of the two- and threedimensional diffusion coefficients of the surface molecules falls between 1 and 1.5 in every case, whereas in the bulk liquid phase the two values are identical. Clearly, in the case of isotropic diffusion, i.e., when the molecules move with the same average speed in all directions, the two- and three-dimensional diffusion coefficients are equal. Indeed, in this case, the three-dimensional MSD is 1.5 times larger than the two-dimensional one, and the factor $k$ in the denominator of eq. 1 makes the corresponding diffusion coefficient values equal. On the other hand, if the motion is restricted to a given plane, the two-dimensional diffusion coefficient within this plane is 1.5 times larger than the three-dimensional one (since the off-plane displacement is zero, the two- and three-dimensional MSD values are equal, and 
the value of $k$ in eq. 1 accounts for this factor of 1.5). To demonstrate this, we have selected those water molecules in the bulk liquid phase simulation the motion of which happened to be restricted to a given, arbitrarily chosen planar layer, and calculated the two- and threedimensional diffusion coefficients of these molecules (only while they happened to stay in this planar layer). The width of this planar layer, $d$, has been chosen to be equal to the width of the surface layer of the corresponding interfacial system (see Fig. 2). The obtained $D$ values are also included in Table 2. As is seen, the two-dimensional diffusion coefficient of these molecules is indeed exactly 1.5 times larger than their three-dimensional one in every case.

In the light of this result it is interesting to see that, for the real surface molecules, the two-dimensional diffusion coefficient within the macroscopic plane of the liquid surface is considerably less than 1.5 times larger than the three-dimensional one, although the average width of the surface layer equals to that of the above, arbitrarily chosen planar layer in the bulk. On one hand, the fact that this ratio is much larger than unity is a clear consequence of the liquid surface being a two-dimensional, quasi-planar object. On the other hand, the fact that the ratio of the two diffusion coefficients at the liquid surface is markedly below 1.5, at least in the time interval within which the $M S D$ vs. $t$ data were fitted, indicates that the surface molecules do diffuse also along the macroscopic surface normal axis, although, evidently, in a much smaller extent than within the macroscopic plane of the liquid surface. The presence of this diffusion component along the macroscopic surface normal axis is a clear consequence of the fact that the liquid surface is not planar, instead, it is corrugated by capillary waves, and hence the motion of the surface molecules is not restricted to a macroscopic plane, either. This is illustrated in Figure 6, showing the displacement vector of two schematic surface molecules (shown by dots) along a schematic wavy surface.

In interpreting this result one has to note that the motion of the interfacial water molecules is bound, and hence it is not expected that motion along the macroscopic surface normal axis can contribute to the diffusion coefficient at long times. This is indeed the case here; the one dimensional MSD of the surface molecules along the macroscopic surface normal axis, $X$, starts deviating from linearity above about $7 \mathrm{ps,} \mathrm{its} \mathrm{slope} \mathrm{becomes} \mathrm{close} \mathrm{to}$ zero around $15 \mathrm{ps}$, and reaches a plateau around $25 \mathrm{ps}$ (see Fig. 4.b). This finding indicates that motion along the macroscopic surface normal axis can only be regarded as a free diffusion up to $7 \mathrm{ps}$, and the aforementioned normal diffusion component caused by the molecular scale roughness of the liquid surface vanishes almost completely beyond about 15 ps. It should be noted that this time scale on which surface molecules do diffuse also along 
the macroscopic surface normal axis agrees well with the mean surface residence time of the molecules (see Table 1). The existence of this normal diffusion component at short times is, not a trivial result, as seen from the difference of the one-dimensional normal MSD data obtained at the liquid surface and in the bulk phase for the molecules that happen to move in a given planar layer for a while. As is seen in Fig. 4.b, in this latter case there is indeed no normal component of the diffusion, as the $M S D$ vs. $t$ data reaches its plateau value already in 2 ps, while its linear part cannot even be seen using our 1 ps resolution, although the width of this planar layer equals, by our definition, to the average width of the interfacial layer.

Finally, it should be noted that the ratio of the two-dimensional diffusion coefficient at the liquid surface and the three-dimensional diffusion coefficient in the bulk liquid phase is resulted in the range of 1.9-2.1 for all water models considered. This is in a reasonable agreement with the finding of Duque et al. for Lennard-Jones fluids, who found values between 2 and 2.4, depending on the temperature of the system. ${ }^{53}$ This agreement indicates that although hydrogen bonding at the water surface does influence the dynamics of the surface molecules, the relation of the (two-dimensional) surface and (three-dimensional) bulk diffusion of the molecules is not sensitive to this hydrogen bonding.

\subsection{Spatial Correlation between Long-Residing Surface Molecules. As we have} already seen, long surface residence time does not correlate at all with the diffusive motion of the surface molecules. To further investigate the question what makes a molecule stay unusually long at the liquid surface, here we analyze whether such long residing molecules are distributed randomly at the liquid surface or their positions are correlated with each other. For this purpose, we have selected the longest-residing $20 \%$ and $10 \%$, respectively, of the surface molecules in each snapshot, projected their positions, represented by those of their $\mathrm{O}$ atoms, to the macroscopic plane of the liquid surface, $Y Z$, and performed a Voronoi analysis on these projections.

Voronoi analysis ${ }^{77,78}$ is a very convenient tool for investigating the presence and extent of local density fluctuations, in other words, the question whether the position of a set of spatial (or planar) points are correlated or uncorrelated with each other. ${ }^{79}$ In a twodimensional set of seeds (in our case, projections of the molecules in the $Y Z$ plane) the Voronoi cell of each seed is the locus of planar points that are closer to this seed than to any other one. ${ }^{80}$ In this sense, the Voronoi cell of a seed covers the area $A$ that belongs to this seed rather than to any other one. By construction, Voronoi cells constitute a tessellation that covers the plane without gaps and overlaps. ${ }^{80}$ Given that the seeds are uniformly distributed, 
the area distribution of their Voronoi cells, $P(A)$, follows a Gaussian shape. ${ }^{79}$ However, when the seeds are distributed in a correlated way, i.e., forming dense patches and leaving rather large empty areas between them, the Voronoi cells around these empty domains extend deeply into the nearby empty area, and consequently have rather large areas. In such cases the Voronoi cell area distribution exhibits a long, exponentially decaying tail at the large area side of its peak. $^{79}$

The Voronoi cell area distribution, $P(A)$, obtained for the longest residing $20 \%$ and $10 \%$ of the surface molecules are shown in Figure 7 as obtained in all the three systems considered. As is seen, the obtained distributions cannot be fitted by a Gaussian function in any case, as these distributions are always asymmetric, having a considerably slower decaying tail at their large area side than at small areas. By contrast, the Voronoi cell area distributions obtained by taking all surface molecules into account turned out to be of perfect Gaussian shape in every case (data not shown). To demonstrate the exponential nature of the large area side decay of the tails, the obtained $P(A)$ data are shown on a logarithmic scale in the insets of Fig. 7. To quantify the degree of clustering of the long residing surface molecules, we have evaluated their Kirkwood-Buff integral, $G^{R}=\int_{0}^{R} 2 \pi r(g(r)-1) d r,{ }^{81}$ up to the $R$ value of $25 \AA$. Unfortunately, our system size was not large enough to provide convergent results for $G^{R}$, nevertheless, it turns out that, considering the $20 \%$ and $10 \%$ longest residing surface molecules, the KB integral well exceeds the values of about 15-20 and 35-40, respectively, for all the three water models considered. This result clearly shows that the molecules that will stay long at the liquid surface are not distributed randomly, their positions are correlated with each other, and hence these molecules tend to be located preferentially at the vicinity of each other, forming relatively dense domains of long-residing molecules at the liquid surface, as it is also illustrated in Figure 8.

3.5. Hydrogen Bonding at the Intrinsic Liquid Surface. To investigate the effect of the interface on the dynamics of the hydrogen bonds between the water molecules, we have calculated the mean hydrogen bond lifetime between two interfacial molecules in the three interfacial systems simulated, and, for comparison, also in the bulk liquid phase. Further, to see whether hydrogen bond lifetime is related to the surface residence time of the molecules in any way, the mean lifetime of the hydrogen bonds formed by two surface molecules that are among the $20 \%$ longest residing ones has also been calculated. Two water molecules have been regarded as being hydrogen bonded to each other if the distance of their $\mathrm{O}$ atoms and 
closest O-H pair have been less than $3.35 \AA$ and $2.45 \AA$ (i.e., the first minimum position of the $\mathrm{O}-\mathrm{O}$ and $\mathrm{O}-\mathrm{H}$ radial distribution functions), respectively.

The mean lifetime of the hydrogen bonds, $\tau_{\mathrm{HB}}$, can be determined in a similar way than the mean surface residence time. Namely, the survival probability of the hydrogen bonds, $L_{\mathrm{HB}}(t)$ (i.e., the probability that a hydrogen bond that exists at $t_{0}$ will remain intact up to $t_{0}+t$ ) has to be calculated, and the exponentially decaying function $\exp \left(-t / \tau_{\mathrm{HB}}\right)$ has to be fitted to these $L_{\mathrm{HB}}(t)$ data. For simplicity, in this analysis, similarly to that of the surface residence time, no breaking of the hydrogen bonds was allowed in any single snapshot.

The obtained $L_{\mathrm{HB}}(t)$ data together with the exponentially decaying functions fitted to them are shown in Figure 9; the corresponding $\tau_{\mathrm{HB}}$ values are collected in Table 3 . To emphasize the exponential decay of the obtained $L_{\mathrm{HB}}(t)$ data, the insets of Fig. 9 show them on a logarithmic scale. As is seen, the mean lifetime of the hydrogen bonds between two interfacial molecules is more than an order of magnitude shorter than the mean surface residence time of the water molecules (the corresponding $\tau_{\mathrm{HB}}$ values are also included in Table 1), indicating that hydrogen bonds formed at the liquid surface and in the bulk liquid phase can indeed be distinguished from each other, and hence hydrogen bond lifetime at the water surface can be meaningfully discussed.

The obtained results reveal that the hydrogen bond lifetime is considerably, by about $40-50 \%$ shorter at the liquid surface than in the bulk liquid phase, and also that the hydrogen bonds formed by two long-residing surface molecules live, on average, $20 \%$ longer than those between two interfacial molecules irrespective of their surface residence time. This latter finding, together with the observed clustering of the long-residing surface molecules around each other suggest that unusually long surface residence might well be related to the hydrogen bonding between such molecules. This point will be further investigated in this paper.

The finding that hydrogen bonds live considerably longer in the bulk liquid phase than at the liquid surface should also be discussed in the light of previous experimental and computer simulation results concerning the strength and geometry of interfacial hydrogen bonds. The question whether interfacial hydrogen bonds are stronger or not than thos in the bulk liquid phase is not settled in the literature. Thus, although the majority of the studies reported somewhat stronger hydrogen bonds, on average, at the interface than in the bulk liquid phase, ${ }^{54,56,58}$ they also found that interfacial hydrogen bonds are, on average, slightly longer rather than shorter than the bulk phase ones. ${ }^{54,58}$ This seeming contradiction was resolved considering that the slightly more distant interfacial hydrogen bonded O-O pairs are 
closer to their equilibrium distance than those in the bulk liquid phase. ${ }^{54,58}$ Further, in a combined experimental and computational study, using a polarizable water model Stiopkin et al. found slightly weaker hydrogen bonds at the water surface than in the bulk phase. ${ }^{55}$ Nevertheless, there is a clear consensus in the literature that the strengths of the interfacial and bulk phase hydrogen bonds do not differ much from each other. ${ }^{54,55,58}$ Further, the lifetime of the hydrogen bonds was claimed to be independent from their strength. ${ }^{58,82}$ To investigate this point, we have calculated the mean interaction energy, $\left\langle U_{\mathrm{HB}}^{\text {pair }}\right\rangle$, between two hydrogen bonded interfacial, and between two hydrogen bonded long-residing interfacial molecules, respectively, and also between two water molecules in the bulk. The obtained values are included in Table 3.

As is seen, interfacial hydrogen bonds are somewhat, by about 3-5\% stronger than those in the bulk liquid phase, while long residing interfacial molecules form, on average, only slightly (i.e., by $0.5-1.5 \%$ ) stronger hydrogen bonds with each other than two interfacial molecules irrespective of their surface residence time. This finding indicates that, as expected, the strength of the hydrogen bonds has indeed no impact on their lifetime, in other words, short lifetime of the interfacial hydrogen bonds cannot be explained by their strength. Instead, it is probably related to the enhanced mobility of the interfacial molecules, as seen from their diffusion coefficients (Table 2). Namely, the fact that interfacial molecules form, on average, less hydrogen bonds with their neighbors than bulk phase ones (as in the direction of the opposite phase they do not have hydrogen bonding partners, see Table 4) leads to an enhanced mobility of these molecules (as they are "tethered" by less hydrogen bonds), and to the faster breaking of their existing hydrogen bonds, even if they are, on average, somewhat stronger than in the bulk liquid phase. In other words, the difference between hydrogen bond lifetimes at the water surface and in the bulk liquid phase is related to the difference of their cooperativity rather than of their strength. Thus, the more hydrogen bonds a molecule forms, the lower is the chance that, after breaking one of these hydrogen bonds, the molecule can move away rather than being brought back to its original position by its remaining hydrogen bonds. This explanation is also in accodance with the claim of Nihonyanagi et al. that the molecular structure of the water surface is highly mobile and dynamic. ${ }^{56}$

This explanation is also in accordance with the different relation of the time scales of surface residence and diffusion observed for Lennard-Jones liquids ${ }^{53}$ and water. This difference can again be explained by the effect of hydrogen bonding on surface residence time. Namely, the time scale of surface residence, being practically equal with that of surface 
diffusion in Lennard-Jones systems, ${ }^{53}$ is increased by the fact that extensive hydrogen bonding of the molecules in water and, in particular, the large amount of hydrogen bonds formed between the interfacial molecules slows down their exchange between the liquid surface and the bulk liquid phase.

Finally, to investigate the difference in the hydrogen bonding pattern around longresiding and non long-residing surface molecules, we have calculated the mean number of hydrogen bonded neighbors, $<n_{\mathrm{HB}}>$, of all interfacial molecules as well as of the longest residing $20 \%$ of them, and also the mean number of hydrogen bonded neighbors of these molecules within the surface layer and outside the surface layer, respectively. For comparison, the mean number of hydrogen bonded neighbors of the molecules in bulk liquid water has also been calculated. All these data are collected in Table 4.

As is seen, long-residing interfacial water molecules have, on average, 3-4\% more hydrogen bonded neighbors within the surface layer, but about $25 \%$ less in the subsequent subsurface layer than the average values corresponding to all interfacial molecules irrespective of their surface residence time. This finding indicates that the somewhat longer lifetime of the hydrogen bonds between two long-residing than between two general interfacial molecules is due to the facts that these hydrogen bonds are, on average, slightly stronger than other interfacial hydrogen bonds, and that long residing surface molecules have, on average, more hydrogen bonded neighbors (i.e., they are tethered more) within the surface layer than other ones. It is also expected ${ }^{83}$ that interfacial molecules having more hydrogen bonded neighbors within the surface layer than the average value are distributed in a correlated way. More importantly, this finding reveals that long surface residence time is related to the number of off-layer hydrogen bonded neighbors, namely that those water molecules will stay longer at the liquid surface that are better isolated from the subsurface region of the liquid phase.

\section{Summary and Conclusions}

In this paper we analyzed the dynamic properties of the water molecules at the intrinsic liquid-vapor interface by molecular dynamics simulation and ITIM intrinsic surface analysis using three different water models. Our results revealed that water molecules stay, on average, considerably longer at the liquid surface than the characteristic time of their surface diffusion, in a clear contrast with Lennard-Jones systems, in which these two time scales are roughly equal. ${ }^{53} \mathrm{We}$ also found that diffusion of the interfacial molecules is considerably 
faster than in the bulk liquid phase both in two and three dimensions, and the lifetime of the hydrogen bonds at the liquid surface is also remarkably shorter than in the bulk phase, in spite of the fact that interfacial hydrogen bonds are, on average, somewhat stronger than bulk phase ones. All these findings seem to be related to the fewer number of hydrogen bonded neighbors the water molecules have at the liquid surface than in the bulk liquid phase. As a consequence, interfacial water molecules are tethered by their hydrogen bonded neighbors in a lesser extent than the bulk phase ones, which leads to their enhanced mobility as well as shorter lifetime of their hydrogen bonds.

We also found that the ratio of the two- and three-dimensional diffusion coefficients of the interfacial water molecules falls between 1 and 1.5 (i.e., the values corresponding to isotropic spatial and planar diffusions, respectively) in every case, indicating that, due to the molecular scale roughness of the liquid surface caused by the capillary waves, interfacial water molecules diffuse also along the macroscopic surface normal axis, although, obviously, in a much smaller extent than within the macroscopic plane of the liquid surface, and this perpendicular diffusion is limited in time. Nevertheless, the time scale on which such a perpendicular diffusion occurs is comparable with the mean surface residence time of the molecules.

Long residing molecules have, on average, slightly more hydrogen bonded neighbors within the surface layer, and considerably less outside the surface layer than those staying shorter at the surface. This finding suggests that long surface residence time is originated in the isolation of the molecules from the subsurface region of the liquid. Further, it is also found that long residing molecules are not distributed evenly at the liquid surface, indicating that surface molecules that are strongly isolated from the rest of the liquid phase form patches "floating" at the surface of liquid water.

Acknowledgements. This work has been supported by the Hungarian OTKA Foundation under Project Nos. 104234 and 119732. G.H. acknowledges the help of Lajos Höfler (Budapest University of Technology and Economics) with supplementary lattice diffusion simulations. The authors are grateful to Marcello Sega (University of Vienna) for useful discussions and critical comments concerning this study. 


\section{References}

(1) Shen, Y. R. The Principles of Nonlinear Optics, Wiley-Interscience: New York, 1984.

(2) Shen, Y. R. Surface Properties Probed by Second-Harmonic and Sum-Frequency Generation. Nature, 1989, 337, 519-525.

(3) Richmond, G. L. Molecular Bonding and Interactions at Aqueous Surfaces as Probed by Vibrational Sum Frequency Spectroscopy. Chem. Rev. 2002, 102, 2693-2724.

(4) Franken, P.; Hill, A.; Peters, C.; Weinreich, G. Generation of Optical Harmonics. Phys. Rev. Letters 1961, 7, 118-120.

(5) Bloembergen, N.; Pershan, P. S. Light Waves at Boundary of Nonlinear Media. Phys. Rev. 1962, 128, 606-623.

(6) Eisenthal, K. B. Liquid Interfaces Probed by Second-Harmonic and Sum-Frequency Spectroscopy. Chem. Rev. 1996, 96, 1343-1360.

(7) Daillant, J.; Gibaud, A. X-Ray and Neutron Reflectivity: Principles and Applications, Springer: Berlin, 1999.

(8) Tolan, M.; X-Ray Scattering from Soft-matter Thin Films, Springer: Berlin, 1999.

(9) Penfold, J. Neutron Reflectivity and Soft Condensed Matter. Curr. Opin. Colloid Interface Sci. 2002, 7, 139-147.

(10) Jähnig, F., Structural Order of Lipids and Proteins in Membranes: Evaluation of Fluorescence Anisotropy Data. Proc. Natl. Acad. Sci. USA 1979, 76, 6361-6365.

(11) Cross, A. J.; Fleming, G. R. Analysis of Time-Resolved Fluorescence Anisotropy Decays. Biophys. J. 1984, 46, 45-56.

(12) Allen, M. P.; Tildesley, D. J. Computer Simulation of Liquids; Clarendon Press: Oxford, 1987.

(13) Pártay, L. B.; Hantal, Gy.; Jedlovszky, P.; Vincze, Á.; Horvai, G. A New Method for Determining the Interfacial Molecules and Characterizing the Surface Roughness in Computer Simulations. Application to the Liquid-Vapor Interface of Water. J. Comp. Chem. 2008, 29, 945-956.

(14) Hantal, Gy.; Darvas, M.; Pártay, L. B.; Horvai, G.; Jedlovszky, P. Molecular Level Properties of the Free Water Surface and Different Organic Liquid/Water Interfaces, as Seen from ITIM Analysis of Computer Simulation Results. J. Phys.: Condens. Matter 2010, 22, 284112-1-14. 
(15) Pártay, L. B.; Jedlovszky, P.; Vincze, Á.; Horvai, G. Properties of Free Surface of Water-Methanol Mixtures. Analysis of the Truly Interfacial Molecular Layer in Computer Simulation. J. Phys. Chem. B. 2008, 112, 5428-5438.

(16) Pártay, L. B.; Jedlovszky, P.; Horvai, G. Structure of the Liquid-Vapor Interface of Water-Acetonitrile Mixtures As Seen from Molecular Dynamics Simulations and Identification of Truly Interfacial Molecules Analysis. J. Phys. Chem. C. 2009, 113, 18173-18183.

(17) Fábián, B.; Szőri, M.; Jedlovszky, P. Floating Patches of HCN at the Surface of Their Aqueous Solutions - Can They Make "HCN World" Plausible? J. Phys. Chem. C 2014, $118,21469-21482$.

(18) Pártay, L. B.; Horvai, G.; Jedlovszky, P. Temperature and Pressure Dependence of the Properties of the Liquid-Liquid Interface. A Computer Simulation and Identification of the Truly Interfacial Molecules Investigation of the Water-Benzene System. $J$. Phys. Chem. C. 2010, 114, 21681-21693.

(19) Linse, P. Monte Carlo Simulation of Liquid-Liquid Benzene-Water Interface. J. Chem. Phys. 1987, 86, 4177-4187.

(20) Benjamin, I. Theoretical Study of the Water/1,2-Dichloroethane Interface: Structure, Dynamics, and Conformational Equilibria at the Liquid-Liquid Interface. J. Chem. Phys. 1992, 97, 1432-1445.

(21) Benjamin, I. Structure, Thermodynamics, and Dynamics of the Liquid/Vapor Interface of Water/Dimethylsulfoxide Mixtures. J. Chem. Phys. 1999, 110, 8070-8079.

(22) Michael, D.; Benjamin, I. Molecular Dynamics Computer Simulations of Solvation Dynamics at Liquid/Liquid Interfaces. J. Chem. Phys. 2001, 114, 2817-2824.

(23) Jorge, M.; Cordeiro, M. N. D. S. Intrinsic Structure and Dynamics of the Water/Nitrobenzene Interface. J. Phys. Chem. C. 2007, 111, 17612-17626.

(24) Jorge, M.; Cordeiro, M. N. D. S. Molecular Dynamics Study of the Interface between Water and 2-Nitrophenyl Octyl Ether. J. Phys. Chem. B 2008, 112, 2415-2429.

(25) Pandit, S. A.; Bostick, D.; Berkowitz, M. L. An Algorithm to Describe Molecular Scale Rugged Surfaces and its Application to the Study of a Water/Lipid Bilayer Interface. J. Chem. Phys. 2003, 119, 2199-2205.

(26) Chacón, E.; Tarazona, P. Intrinsic Profiles beyond the Capillary Wave Theory: A Monte Carlo Study. Phys Rev. Letters 2003, 91, 166103-1-4

(27) Chowdhary, J.; Ladanyi, B. M. Water-Hydrocarbon Interfaces: Effect of Hydrocarbon Branching on Interfacial Structure. J. Phys. Chem. B. 2006, 110, 15442-15453. 
(28) Jorge, M.; Jedlovszky, P.; Cordeiro, M. N. D. S. A Critical Assessment of Methods for the Intrinsic Analysis of Liquid Interfaces. 1. Surface Site Distributions. J. Phys. Chem. C. 2010, 114, 11169-11179.

(29) Mezei, M. A New Method for Mapping Macromolecular Topography. J. Mol. Graphics Modell. 2003, 21, 463-472.

(30) Wilard, A. P.; Chandler, D. Instantaneous Liquid Interfaces. J. Phys. Chem. B. 2010, 114, 1954-1958.

(31) Sega, M.; Kantorovich, S.; Jedlovszky, P; Jorge, M. The Generalized Identification of Truly Interfacial Molecules (ITIM) Algorithm for Nonplanar Interfaces. J. Chem. Phys. 2013, 138, 044110-1-10.

(32) Jorge, M.; Hantal, G.; Jedlovszky, P.; Cordeiro, M. N. D. S. A Critical Assessment of Methods for the Intrinsic Analysis of Liquid Interfaces: 2. Density Profiles. J. Phys. Chem. C. 2010, 114, 18656-18663.

(33) Sega, M.; Fábián, B.; Jedlovszky, P. Layer-by-Layer and Intrinsic Analysis of Molecular and Thermodynamic Properties across Soft Interfaces. J. Chem. Phys. 2015, 143, 114709-1-8.

(34) Darvas, M.; Jorge, M.; Cordeiro, M. N. D. S.; Kantorovich, S. S.; Sega, M.; Jedlovszky, P. Calculation of the Intrinsic Solvation Free Energy Profile of an Ionic Penetrant Across a Liquid/Liquid Interface with Computer Simulations. J. Phys. Chem. B 2013, 117, 16148-16156.

(35) Bresme, F.; Chacón, E.; Tarazona, P.; Wynveen, A. The Structure of Ionic Aqueous Solutions at Interfaces: An Intrinsic Structure Analysis. J. Chem. Phys. 2012, 137, 114706-1-10.

(36) Sega, M.; Horvai, G.; Jedlovszky, P. Microscopic Origin of the Surface Tension Anomaly of Water. Langmuir 2014, 30, 2969-2972.

(37) Sega, M.; Horvai, G.; Jedlovszky, P. Two-Dimensional Percolation at the Free Water Surface and its Relation with the Surface Tension Anomaly of Water. J. Chem. Phys. 2014, 141, 054707-1-11.

(38) Bresme, F.; Chacón, E.; Martínez, H.; Tarazona, P. Adhesive Transitions in Newton Black Films: A Computer Simulation Study. J. Chem. Phys. 2011, 134, 214701-1-12.

(39) Hantal, G.; Cordeiro, M. N. D. S.; Jorge, M. What Does an Ionic Liquid Surface Really Look Like? Unprecedented Details from Molecular Simulations. Phys. Chem. Chem. Phys. 2011, 13, 21230-21232. 
(40) Lísal, M.; Posel, Z.; Izák, P. Air-Liquid Interfaces of Imidazolium-Based $\left[\mathrm{TF}_{2} \mathrm{~N}^{-}\right]$ Ionic Liquids: Insight from Molecular Dynamics Simulations. Phys. Chem. Chem. Phys. 2012, 14, 5164-5177.

(41) Hantal, G.; Voroshylova, I.; Cordeiro, M. N. D. S.; Jorge, M. A Systematic Molecular Simulation Study of Ionic Liquid Surfaces Using Intrinsic Analysis Methods. Phys. Chem. Chem. Phys. 2012, 14, 5200-5213.

(42) Lísal, M.; Izák, P. Molecular Dynamics Simulations of n-Hexane at 1-Butyl-3Methylimidazolium bis(Trifluoromethylsulfonyl) Imide Interface. J. Chem. Phys. 2013, 139, 014704-1-15.

(43) Bresme, F.; Chacón, E.; Tarazona, P.; Tay, K. Intrinsic Structure of Hydrophobic Surfaces: The Oil-Water Interface. Phys Rev. Letters 2008, 101, 056102-1-4.

(44) Abrankó-Rideg, N.; Darvas, M.; Horvai, G.; Jedlovszky, P. Immersion Depth of Surfactants at the Free Water Surface: A Computer Simulation and ITIM Analysis Study. J. Phys. Chem. B 2013, 117, 8733-8746.

(45) Lindahl, E; Edholm, O. Solvent Diffusion Outside Macromolecular Surfaces. Phys. Rev. E 1998, 57, 791-796.

(46) Åman, K.; Lindahl, E.; Edholm, O.; Håkansson, P.; westlund, P. O. Structure and Dynamics of Interfacial Water in an $L_{\alpha}$ Phase Lipid Bilayer from Molecular Dynamics Simulations. Biophys. J. 2003, 84, 102-115.

(47) Liu, P.; Harder, E.; Berne, B. On the Calculation of Diffusion Coefficientsin Confined Fluids and Interfaces with an Application to the Liquid-Vapor Interface of Water. $J$. Phys. Chem. B 2004, 108, 6595-6602.

(48) Bühn, J. B.; Bopp, P. A.; Hampe, M. J. A Molecular Dynamics Study of the LiquidLiquid Interface: Structure and Dynamics. Fluid Phase Equilib. 2004, 224, 221-230.

(49) Sega, M.; Vallauri, R.; Melchionna, S. Diffusion of Water in Confined Geometry: The Case of a Multilamellar Bilayer. Phys. Rev. E 2005, 72, 041201-1-4.

(50) Bhide, S. Y.; Berkowitz, M. L. Structure and Dynamics of Water at th Interface with Phospholipid Bilayers. J. Chem. Phys. 2005, 123, 224702-1-16.

(51) Benjamin, I. Reactivity and Dynamics at Liquid Interfaces. In: Reviews in Computational Chemistry, Parrill, A. L.; Lipkowitz, K. B., Eds.; Wiley: Chichester, 2015; Vol. 28, pp. 205-313.

(52) Chowdhary, J.; Ladanyi, B. M. Water-Hydrocarbon Interfaces: Effect of Hydrocarbon Branching on Single-Molecule Relaxation. J. Phys. Chem. B. 2008, 112, 6259-6273. 
(53) Duque, D.; Tarazona, P.; Chacón, E. Diffusion at the Liquid-Vapor Interface. J. Chem. Phys. 2008, 128, 134704-1-10.

(54) Jedlovszky, P. The Hydrogen Bonding Structure of Water at the Vicinity of Apolar Interfaces. A Computer Simulation Study. J. Phys.: Condens. Matter 2004, 16, S5389S5402.

(55) Stiopkin, I. V.; Weeraman, C.; Pieniazek, P. A.; Shalhout, F. Y.; Skinner, J. L.; Benderskii, A. V. Hydrogen Bonding at the Water Surface Revealed by Isotopic Dilution Spectroscopy. Nature 2011, 474, 192-195.

(56) Nihonyanagi, S.; Ishiyama, T.; Lee, T. K.; Yamaguchi, S.; Bonn, M.; Morita, A.; Tahara, T. Unified Molecular View of the Air/Water Interface Based on Experimental and Theoretical $\chi^{(2)}$ Spectra of an Isotopically Diluted Water Surface. J. Am. Chem. Soc. 2011, 133, 16875-16880.

(57) Skinner, J. L.; Pieniazek, P. A.; Gruenbaum, S. M. Vibrational Spectroscopy of Water at Interfaces. Acc. Chem. Res. 2012, 45, 93-100.

(58) Vila Verde, A.; Bolhuis, P. G.; Campen, R. K. Statics and Dynamics of Free and Hydrogen Bonded OH Groups at the Air/Water Interface. J. Phys. Chem. B. 2012, 116, 9467-9481.

(59) Du, Q.; Freysz, E.; Shen, Y. R. Surface Vibrational Spectroscopic Studies of Hydrogen Bonding and Hydrophobicity, Science 1994, 264, 826-828.

(60) Gragson, D. E.; Richmond, G. L. Investigations of the Structure and Hydrogen Bonding of Water Molecules at Liquid Surfaces by Vibrational Sum Frequency Spectroscopy. J. Phys. Chem. B. 1998, 102, 3847-3861.

(61) Morita, A.; Hynes, J. T. A Theoretical Analysis of the Sum Frequency Generation Spectrum of the Water Surface. Chem. Phys. 2000, 258, 371-390.

(62) Buch, V. Molecular Structure and OH-Stretch Spectra of Liquid Water Surface. J. Phys. Chem. B. 2005, 109, 17771-17774.

(63) Auer, B. M.; Skinner, L. J. IR and Raman Spectra of Liquid water: Theory and Interpretation. J. Chem. Phys. 2008, 128, 224511-1-12.

(64) Fan, Y.; Chen, X.; Yang, L.; Cremer, P. S.; Gao, Y. Q. On the Structure of Water at the Aqueous/Air Interface. J. Phys. Chem. B. 2009, 113, 11672-11679.

(65) Berendsen, H. J. C.; Grigera, J. R.; Straatsma, T. The Missing Term in Effective pair Potentials. J. Phys. Chem. 1987, 91, 6269-6271. 
(66) Jorgensen, W. L.; Chandrashekar, J.; Madura, J. D.; Impey, R.; Klein, M. L. Comparison of Simple Potential Functions for Simulating Liquid Water. J. Chem. Phys. 1983, 79, 926-935.

(67) Rick, S. W. A Reoptimization of the Five-Site Water Potential (TIP5P) for Use with Ewald Sums. J. Chem. Phys. 2004, 120, 6085-6093.

(68) Hess, B.; Kutzner, C.; van der Spoel, D.; Lindahl, E. GROMACS 4: Algorithms for Highly Efficient, Load-Balanced, and Scalable Molecular Simulation. J. Chem. Theory Comput. 2008, 4, 435-447.

(69) Hess, B. P-LINCS: A Parallel Linear Constraint Solver for Molecular Simulation. J. Chem. Theory Comput. 2008, 4, 116-122.

(70) Nosé, S. A Molecular Dynamics Method for Simulations in the Canonical Ensemble. Mol. Phys. 1984, 52, 255-268.

(71) Hoover, W. G. Canonical Dynamics: Equilibrium Phase-Space Distributions. Phys. Rev. A 1985, 31, 1695-1697.

(72) Essman, U.; Perera, L.; Berkowitz, M. L.; Darden, T.; Lee, H.; Pedersen, L. G. A Smooth Particle Mesh Ewald Method. J. Chem. Phys. 1995, 103, 8577-8594.

(73) Parrinello, M.; Rahman, A. Polymorphic Transitions in Single Crystals: A New Molecular Dynamics Method. J. Appl. Phys. 1981, 52, 7182-7190.

(74) Haar, L.; Gallagher, J. S.; Kell, G. S. NBS/NRC Steam Tables, Hemisphere Press: New York, 1984.

(75) Rideg, N. A.; Darvas, M.; Varga, I.; Jedlovszky, P. Lateral Dynamics of Surfactants at the Free Water Surface. A Computer Simulation Study. Langmuir 2012, 28, 1494414953.

(76) Chowdhary, J.; Ladanyi, B. M. Surface Fluctuations at the Liquid-Liquid Interface. Phys. Rev. E 2008, 77, 031609.

(77) Okabe, A.; Boots, B.; Sugihara, K.; Chiu, S. N. Spatial Tessellations: Concepts and Applications of Voronoi Diagrams, John Wiley: Chichester, 2000.

(78) Medvedev, N. N. The Voronoi-Delaunay Method in the Structural Investigation of Non-Crystalline Systems, SB RAS: Novosibirsk, 2000, in Russian.

(79) Zaninetti, L. The Voronoi Tessalation Generated from Different Distributions of Seeds. Phys. Lett. A 1992, 165, 143-147.

(80) Voronoi, G. F. Recherches sur le Paralléloèders Primitives. J. Reine Angew. Math. 1908, 134, 198-287. 
(81) Krüger, P.; Schnell, S. K.; Bedeaux, D.; Kjelstrup, S.; Vlugt, T. J. H.; Simon, J. M. Kirkwood-Buff Integrals for Finite Volumes. J. Phys. Chem. Letters 2013, 4, 235-238.

(82) Laage, D.; Hynes, J. T. Do More Strongly Hydrogen-Bonded Water Molecules Reorient More Slowly? Chem. Phys. Letters 2006, 433, 80-85.

(83) Stanley, H. E.; Teixeira, J. Interpretation of the Unusual behavior of $\mathrm{H}_{2} \mathrm{O}$ and $\mathrm{D}_{2} \mathrm{O}$ at Low Temperatures Tests of a percolation Model. J. Chem. Phys. 1980, 73, 3404-3422. 


\section{Tables}

TABLE 1. Characteristic Times of Various Processes Studied at the Surface of Liquid Water (in ps Units). Error Bars Are Below 1\% in Every Case.

\begin{tabular}{cccc}
\hline \hline system & $\tau_{\text {surf }}$ & $\tau_{\mathrm{D}}$ & $\tau_{\mathrm{HB}}$ \\
\hline SPC/E & 14.5 & 4.24 & 1.11 \\
TIP4P & 11.4 & 2.90 & 0.79 \\
TIP5P-E & 12.1 & 3.02 & 0.69 \\
\hline \hline
\end{tabular}

TABLE 2. Diffusion Coefficients of Water Obtained at the Liquid Surface and in the Bulk Liquid Phase in Two and Three Dimensions (in $\AA^{2} / p s$ Units)

\begin{tabular}{lllll}
\hline \hline & & SPC/E & TIP4P & TIP5P-E \\
\hline \multirow{3}{*}{3 dimensions } & surface, all molecules & 0.357 & 0.536 & 0.505 \\
& surface, longest residing 20\% & 0.360 & 0.523 & 0.502 \\
& surface, longest residing 10\% & 0.351 & 0.519 & 0.502 \\
& bulk liquid phase & 0.262 & 0.365 & 0.335 \\
& bulk, moving in an arbitrary plane & 0.156 & 0.231 & 0.172 \\
& surface, all molecules & 0.501 & 0.744 & 0.710 \\
& surface, longest residing 20\% & 0.508 & 0.748 & 0.708 \\
\cline { 2 - 3 } & surface, longest residing 10\% & 0.499 & 0.748 & 0.710 \\
& bulk liquid phase & 0.262 & 0.366 & 0.335 \\
& bulk, moving in an arbitrary plane & 0.235 & 0.348 & 0.259 \\
\hline
\end{tabular}


TABLE 3. Average Lifetime of Hydrogen Bonds and Average Interaction Energy of Hydrogen Bonding Pairs in the Bulk Liquid Phase, at the Liquid-Vapor Interface, and among the Longest Residing $20 \%$ of the Interfacial Molecules

\begin{tabular}{cccccccc}
\hline \hline \multirow{2}{*}{$\begin{array}{c}\text { water } \\
\text { model }\end{array}$} & \multicolumn{3}{c}{$\tau_{\mathrm{HB}} / \mathrm{ps}$} & & \multicolumn{2}{c}{$<U_{\mathrm{HB}}^{\text {pair }}>/ \mathrm{kJ} \mathrm{mol}^{-1}$} \\
\cline { 2 - 3 } & bulk & interface & longest residing & & bulk & interface & longest residing \\
\hline SPC/E & 1.90 & 1.11 & 1.37 & & -18.58 & -19.64 & -19.95 \\
TIP4P & 1.45 & 0.79 & 0.94 & & -16.24 & -16.95 & -17.02 \\
TIP5P-E & 1.54 & 0.69 & 0.81 & & -15.99 & -16.54 & -16.61 \\
\hline \hline
\end{tabular}

TABLE 4. Average Number of Hydrogen Bonded Neighbors in the Bulk Liquid Phase, at the Liquid-Vapor Interface, and among the Longest Residing $20 \%$ of the Interfacial Molecules

\begin{tabular}{ccccccccc}
\hline \hline \multirow{2}{*}{$\begin{array}{c}\text { water } \\
\text { model }\end{array}$} & bulk & \multicolumn{3}{c}{ interfacial } & & \multicolumn{3}{c}{ long residing interfacial } \\
\cline { 3 - 5 } & & total & $\begin{array}{c}\text { within the } \\
\text { surface layer }\end{array}$ & off-layer & & total & $\begin{array}{c}\text { within the } \\
\text { surface layer }\end{array}$ & off-layer \\
\hline SPC/E & 3.80 & 3.31 & 2.43 & 0.88 & & 3.18 & 2.52 & 0.66 \\
TIP4P & 3.74 & 3.23 & 2.31 & 0.92 & & 3.09 & 2.39 & 0.70 \\
TIP5P-E & 3.76 & 3.09 & 2.20 & 0.89 & & 2.93 & 2.28 & 0.65 \\
\hline \hline
\end{tabular}




\section{Figure legend}

Figure 1. Instantaneous equilibrium snapshot of the liquid-vapor interface of SPC/E water, as resulted from our simulation. Water molecules being identified as interfacial are marked by red, non-interfacial water molecules are marked by blue color. $\underline{X}$ is the macroscopic surface normal vector, pointing from the liquid to the vapor phase.

Figure 2. Number density profile of the water molecules across the liquid-vapor interface, as obtained from our simulations with the SPC/E (black solid line), TIP4P (red dashed line) and TIP5P-E (green dash-dot-dotted line) water models. Contribution of the surface layer to these profiles are also indicated (SPC/E: black squares, TIP4P: red circles, TIP5P-E: green triangles). All profiles shown are averaged over the two liquid-vapor interfaces present in the basic box.

Figure 3. Survival probability of the water molecules within the surface layer of the liquid phase, as obtained from the simulations with the SPC/E (black circles), TIP4P (red circles) and TIP5P-E (green circles) water models. The exponentially decaying functions fitted to these data sets above 20 ps are shown by dashed lines. To emphasize the exponential decay of the obtained data sets, the inset shows them on a logarithmic scale.

Figure 4. (a) Mean square displacement of the water molecules as obtained for the SPC/E model at the surface layer (black square), in the bulk liquid phase (blue down triangles), and only for those molecules in the bulk liquid phase that happen to move within a given plane (purple diamonds). The inset shows the mean square displacement of all surface molecules (squares) as well as that of the longest residing 20\% (circles) and 10\% (triangles) of them. Open symbols correspond here to three dimensional diffusion, while filled symbols correspond to diffusion in a given plane (i.e., in the macroscopic plane of the surface, $Y Z$, in the case of the interfacial molecules, and in an arbitrary plane in the case of the bulk phase molecules). (b) Mean square displacement of the water molecules as obtained for the SPC/E model in one dimension at the liquid surface for all surface molecules (black squares) as well as for the longest residing 20\% (red circles) and 10\% (green up triangles) of them, in the bulk liquid phase along an arbitrary direction (blue down triangles), and only for those molecules 
in the bulk liquid phase that happen to move within a given plane, along the normal of this plane (purple diamonds).

Figure 5. Trajectory of three long residing surface molecules (filled circles, L1: black, L2: red, L3: blue) and of three surface molecules the surface residence time of which is close to the average value (open circles, A1: green, A2: orange, A3: purple) in the macroscopic plane of the liquid surface, $Y Z$, explored while they belong to the surface layer.

Figure 6. Schematic illustration of the displacement of two interfacial molecules (shown by dots) while they diffuse within the surface layer (arrows), illustrating that, due to the molecular scale roughness of the liquid surface caused by the capillary wave, surface diffusion does have a non-negligible component also along the macroscopic surface normal axis, $X$.

Figure 7. Area distribution of the Voronoi polygons obtained by projecting the centers of the longest residing $20 \%$ (filled circles) and 10\% (open circles) surface molecules, respectively, to the macroscopic plane of the surface, $Y Z$, and constructing the Voronoi tessellation around them. The Gaussian functions best fitting these distributions are shown by red solid curves. To emphasize the exponential decay of the obtained distributions at the large area side of their peak, the insets show the same data sets on a logarithmic scale, where exponential decay turns to a straight line.

Figure 8. Instantaneous snapshots showing the longest residing $20 \%$ (left panel) and $10 \%$ (right panel), respectively, of the surface water molecules from top view in an equilibrium configuration of the interfacial SPC/E system. The position of the molecules is represented by their $\mathrm{O}$ atom, being projected to the macroscopic plane of the surface, $Y Z$.

Figure 9. Survival probability of the hydrogen bonds formed by two water molecules in the bulk liquid phase (blue squares), at the liquid-vapor interface (red circles), as well as by two of the longest residing $20 \%$ of the interfacial water molecules (green triangles), as obtained from the simulations with the SPC/E (top panel), TIP4P (middle panel) and TIP5P-E (bottom panel) water models. The exponentially decaying functions fitted to these data sets are shown by dashed lines. To emphasize the exponential decay of the obtained data sets, the insets show them on a logarithmic scale. 
Figure 1.

Fábián et al.

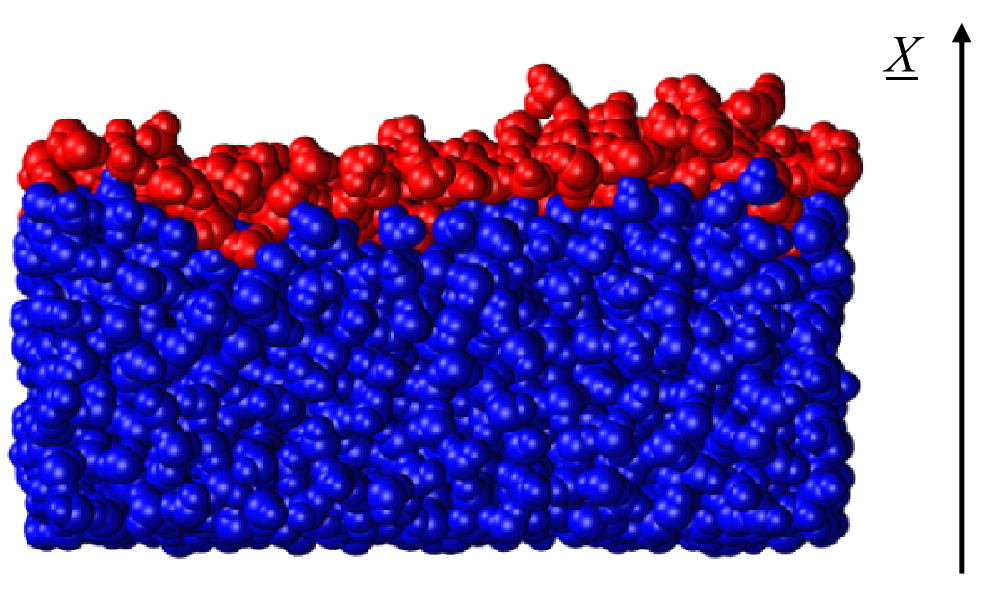


Figure 2.

Fábián et al.

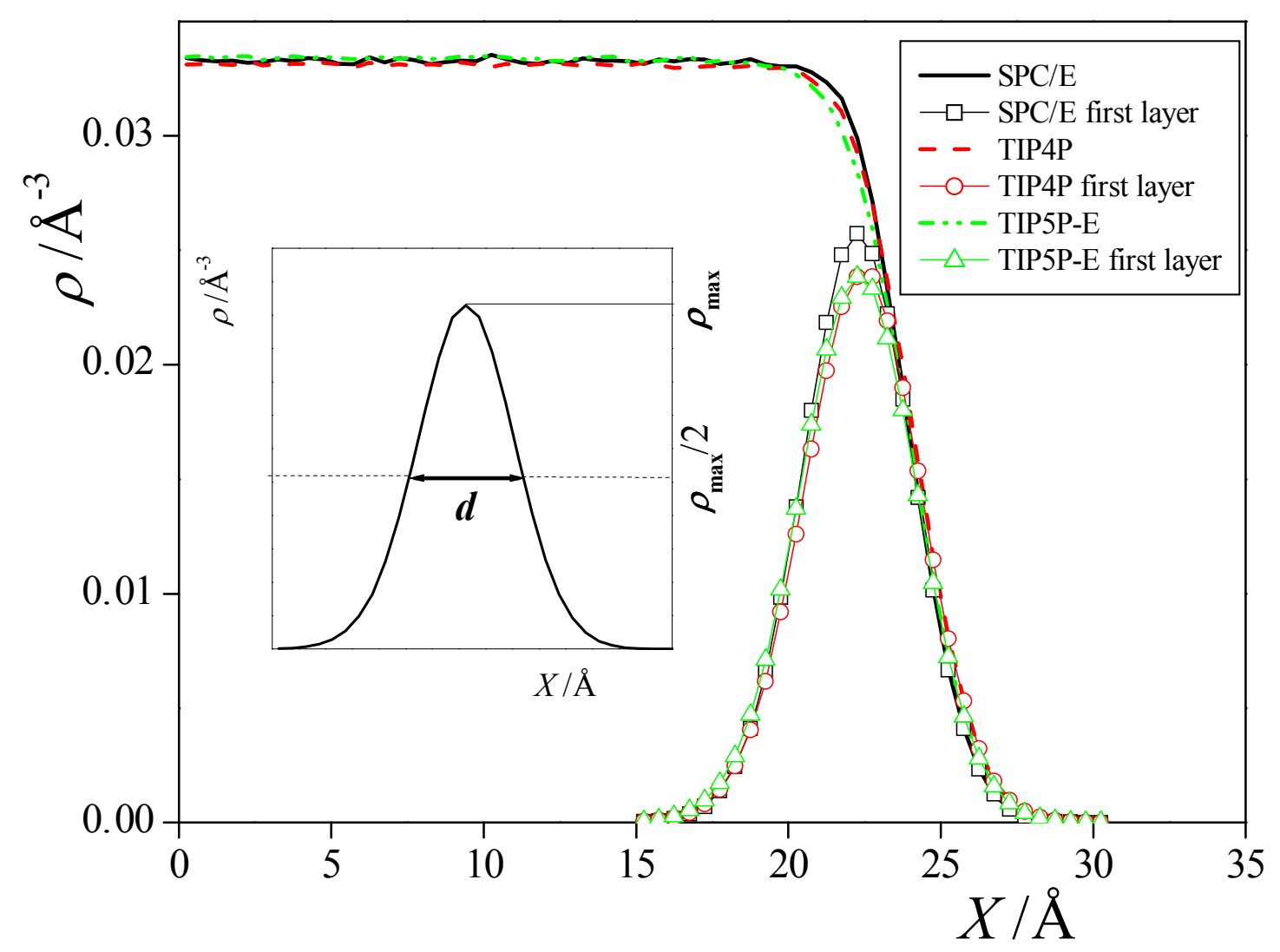


Figure 3.

Fábián et al.

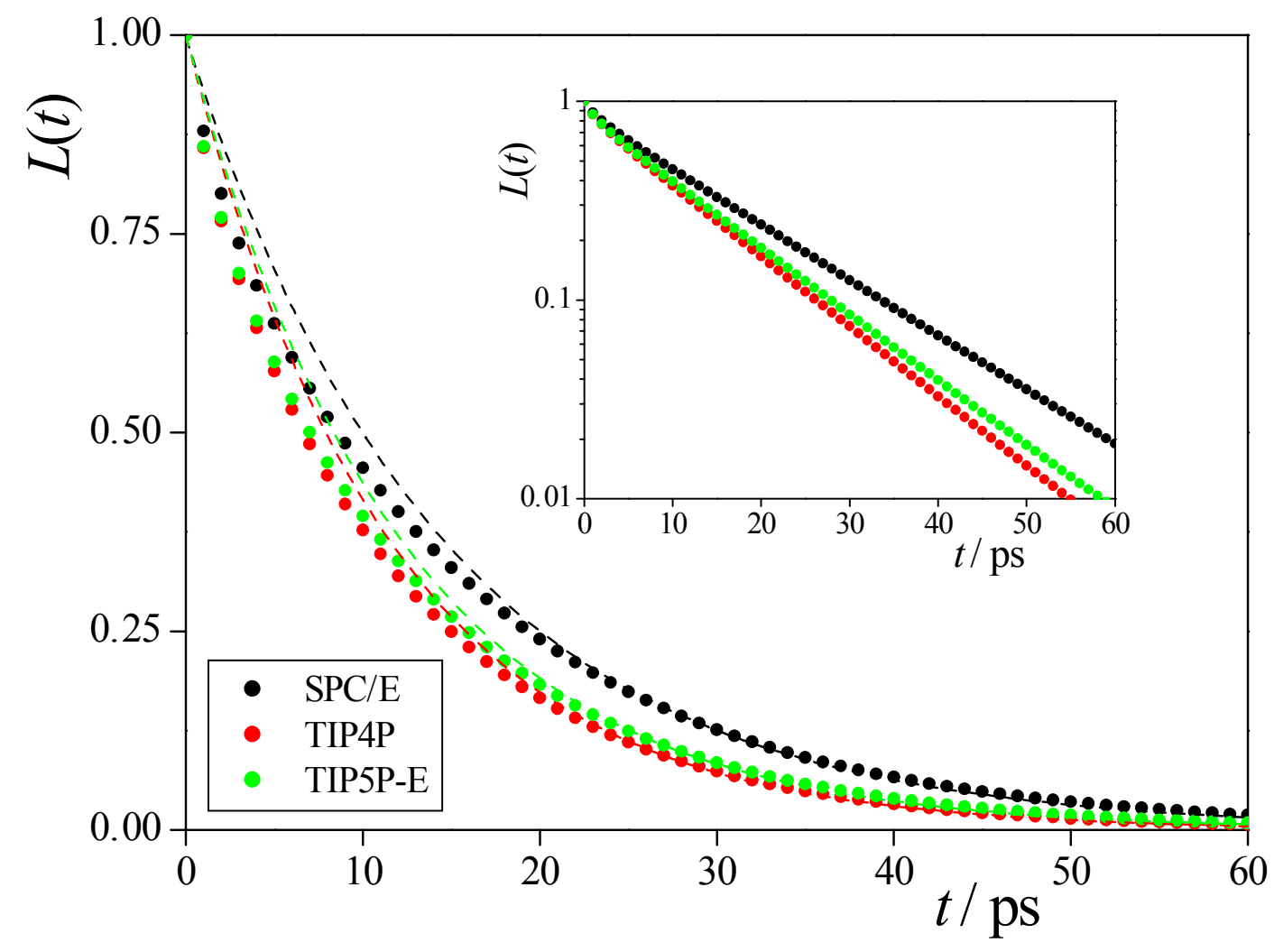


Figure 4.

Fábián et al.

(a)

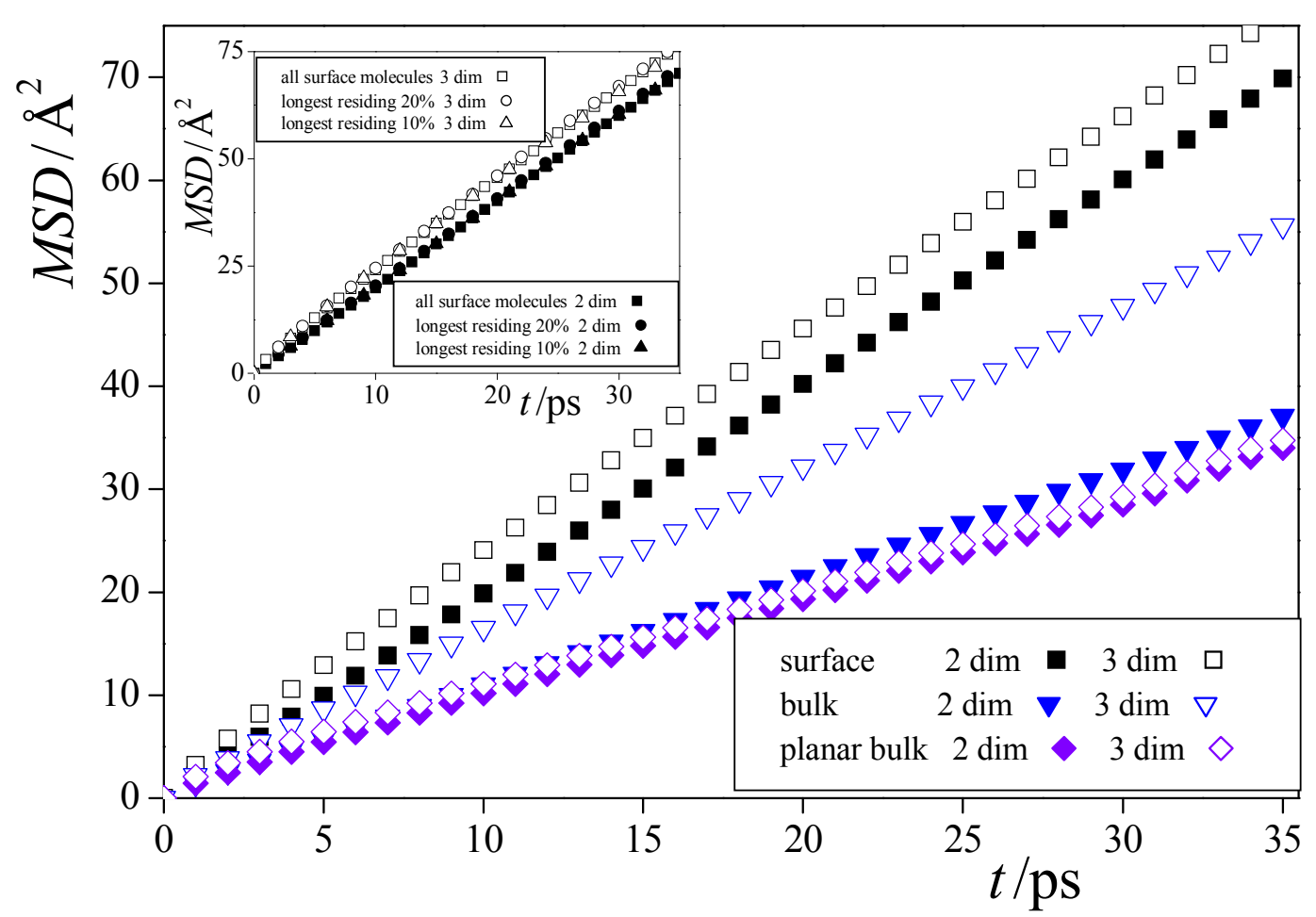

(b)

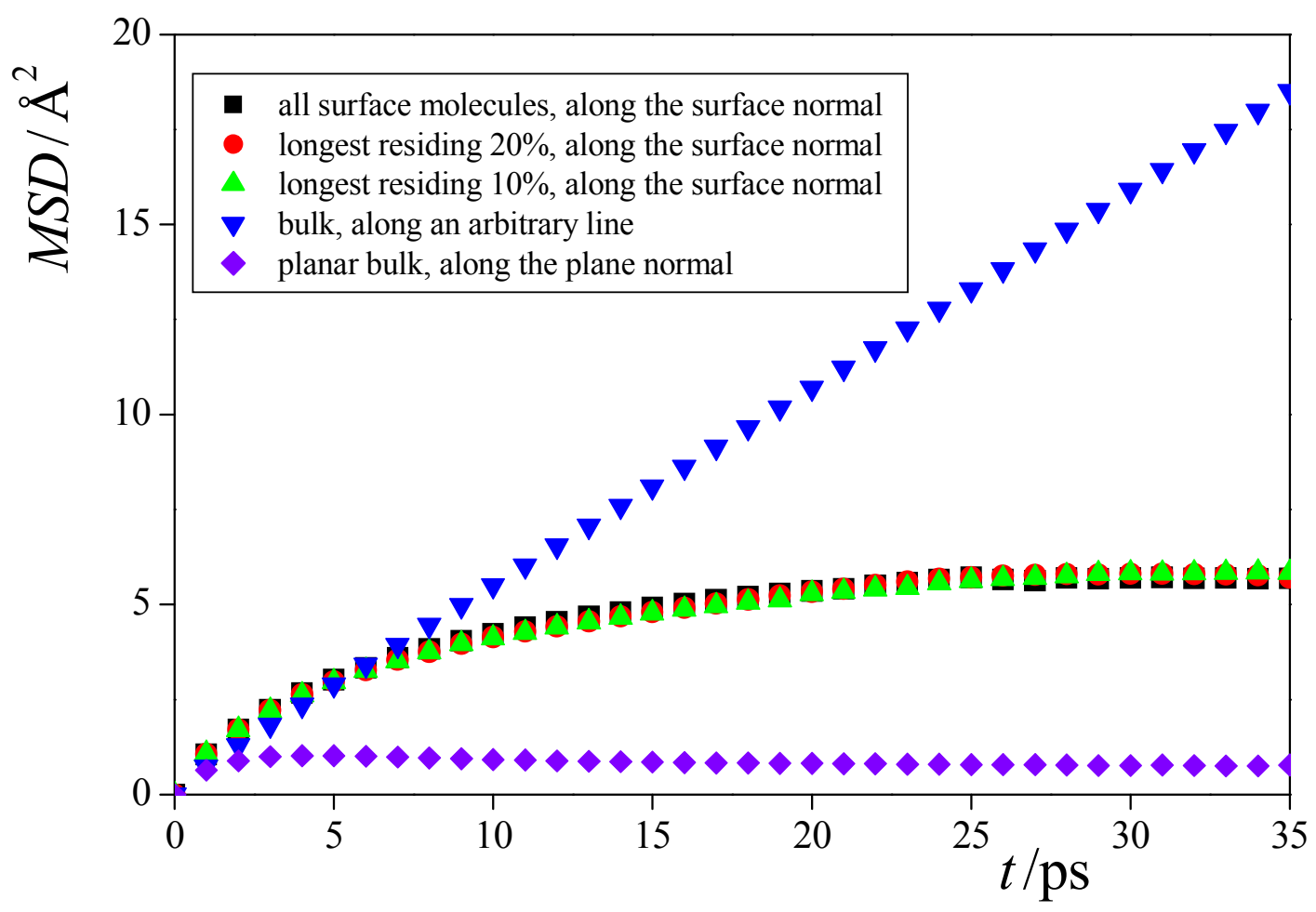

ACS Paragon Plủs Environment 
Figure 5.

Fábián et al.

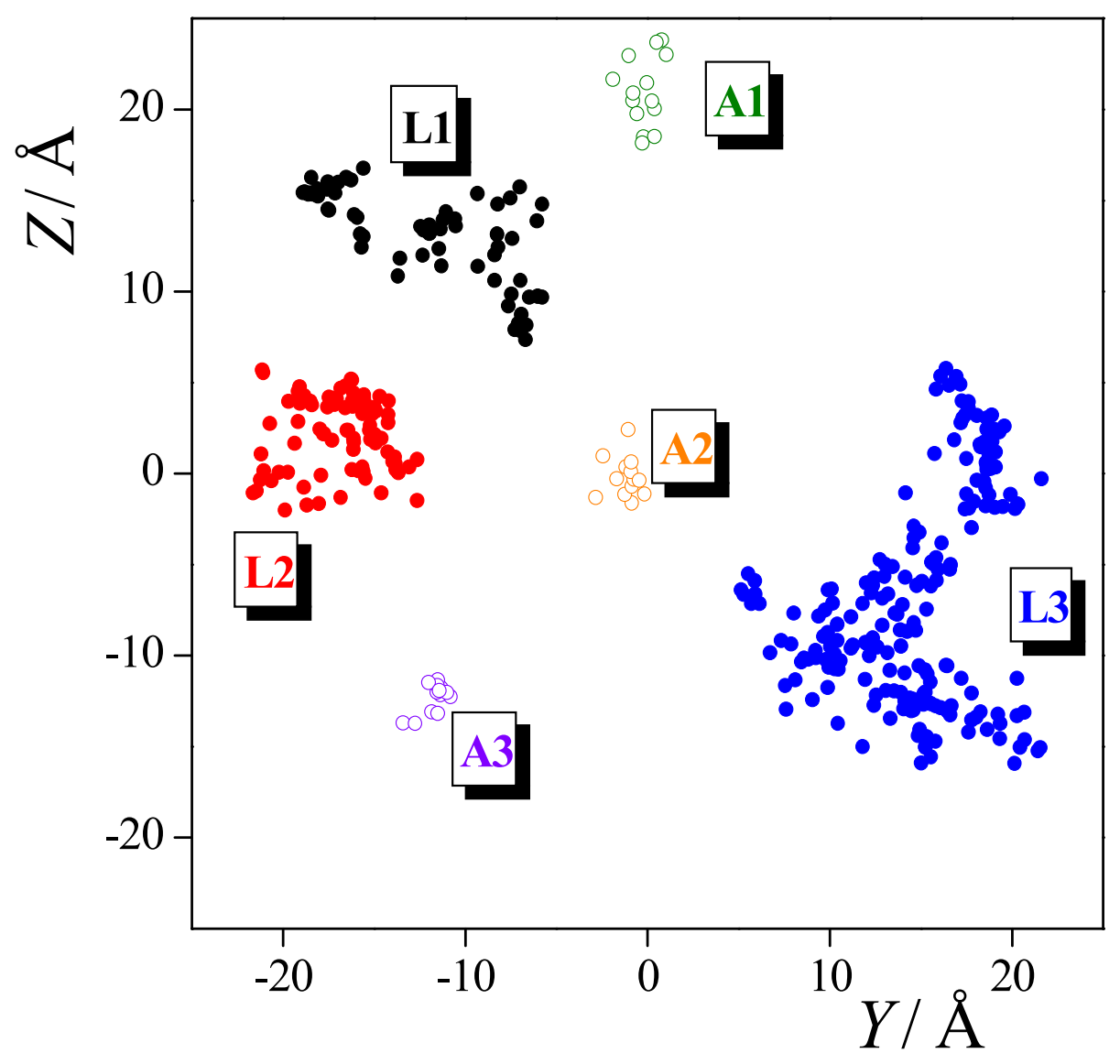


Figure 6.

Fábián et al.

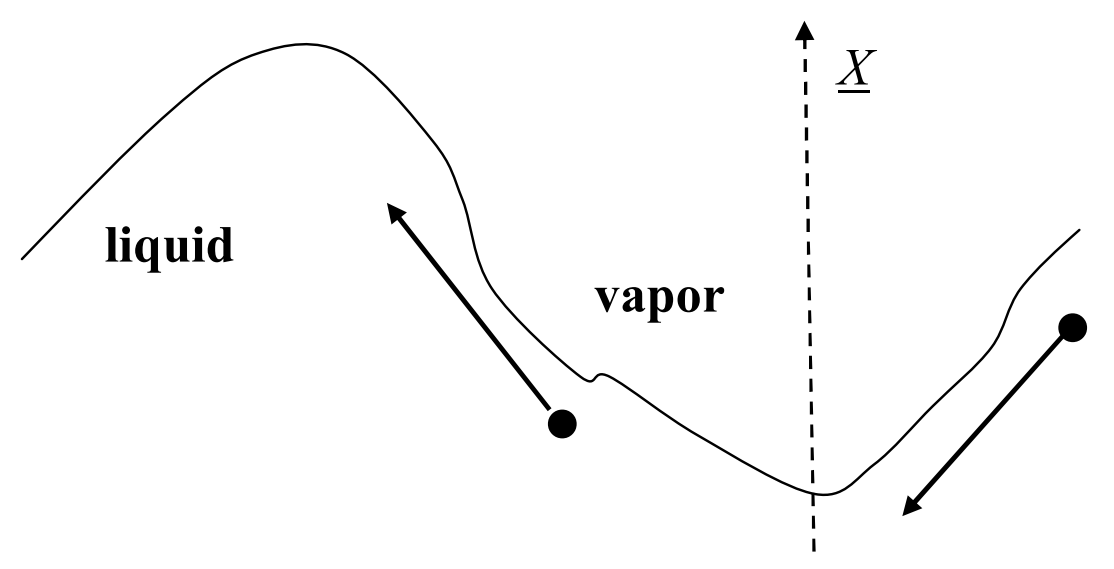


Figure 7.

Fábián et al.

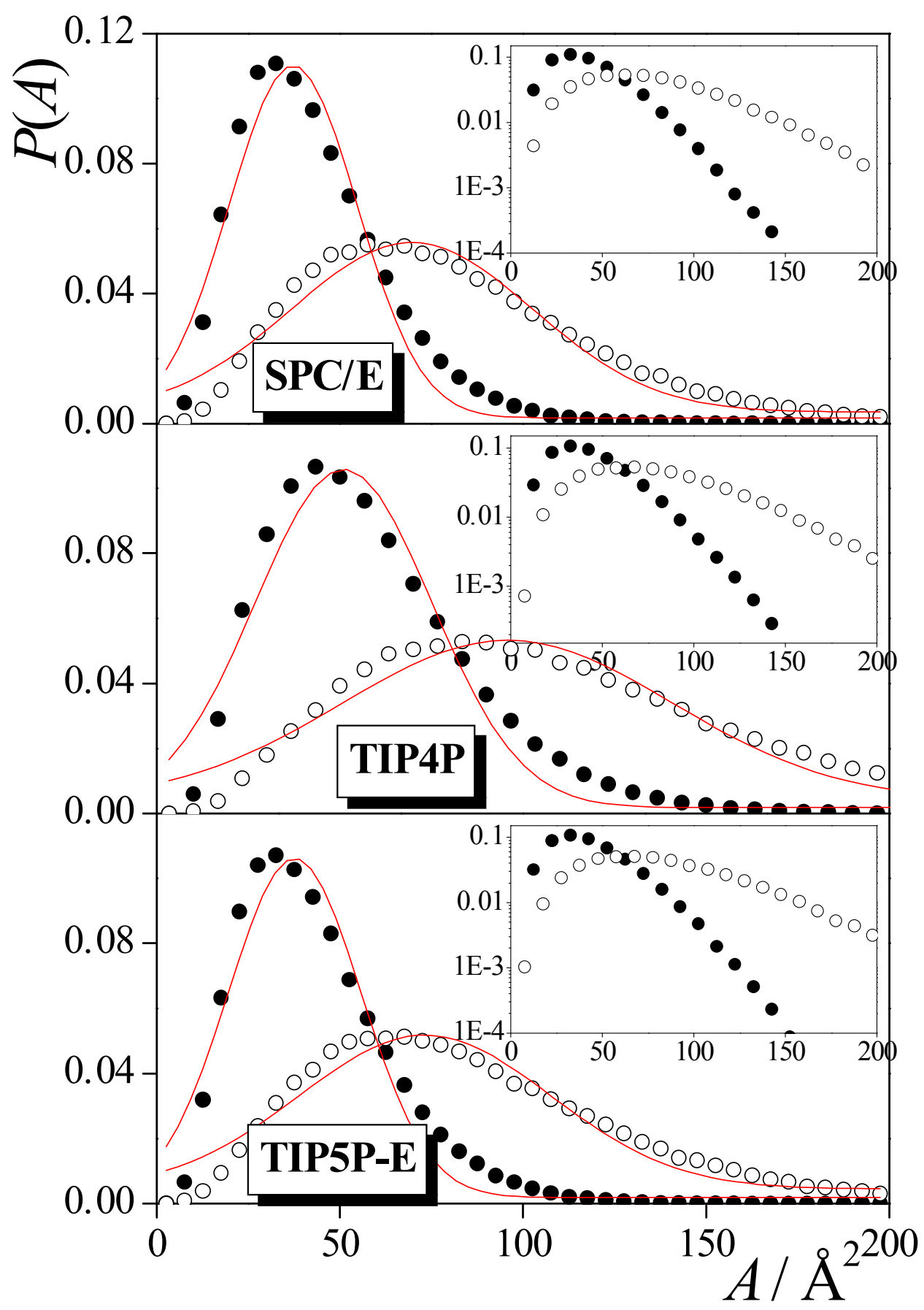


Figure 8.

Fábián et al.
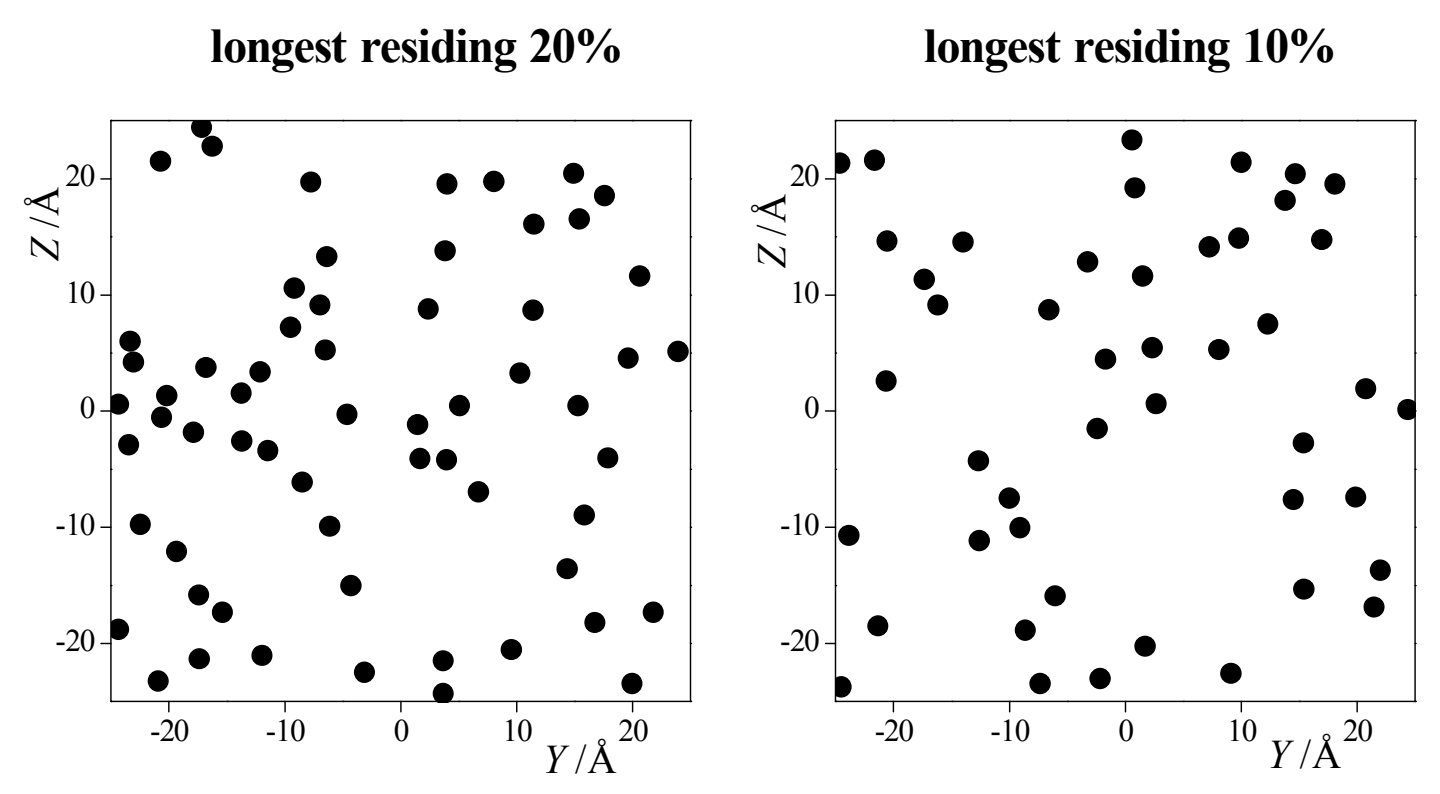

39

40

41

42

43

44

45

46

47

48

49

50

51

52

53

54

55

56

57

58

59

60 
Figure 9.

Fábián et al.

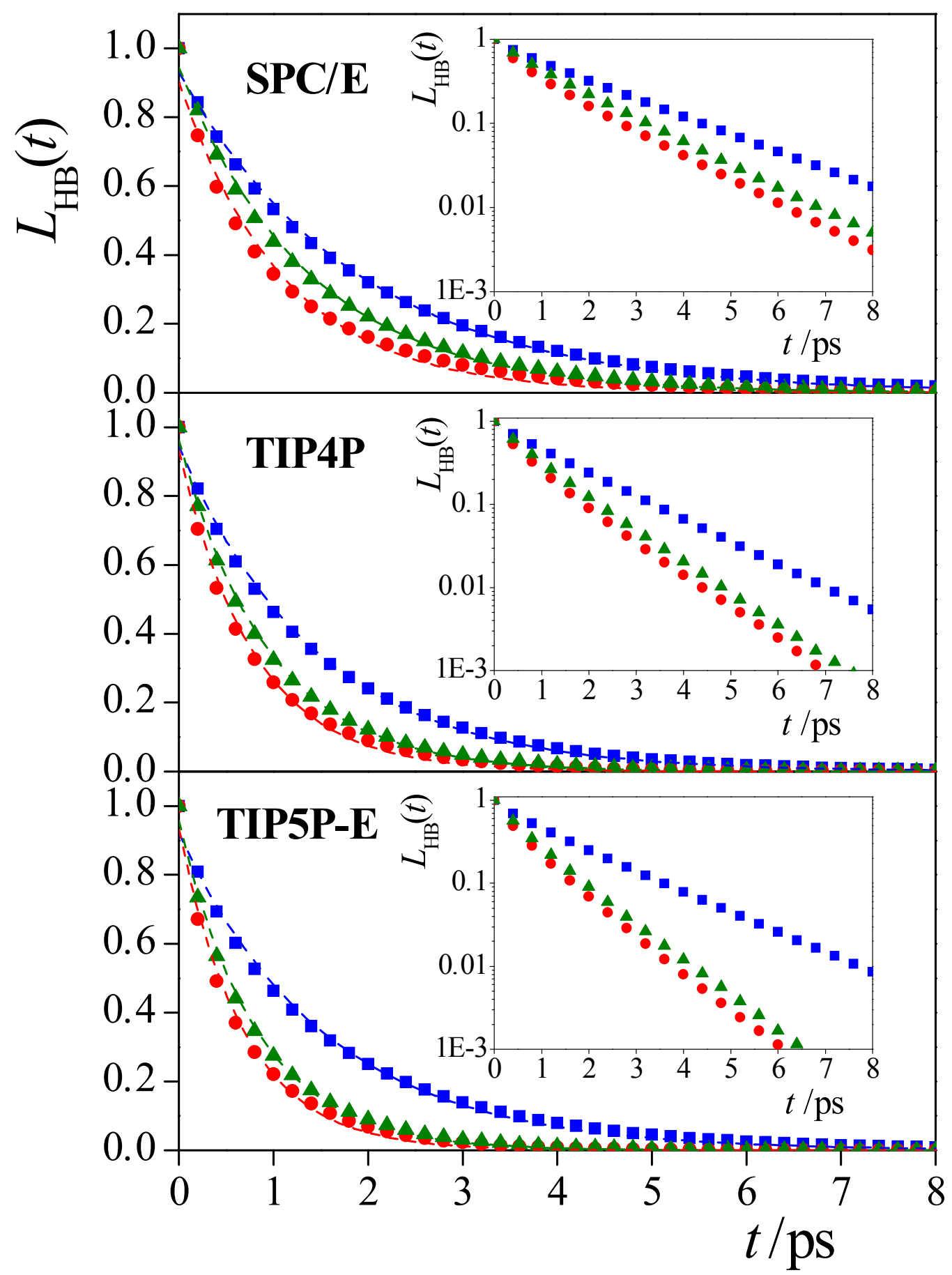




\section{Table of Contents Graphics:}
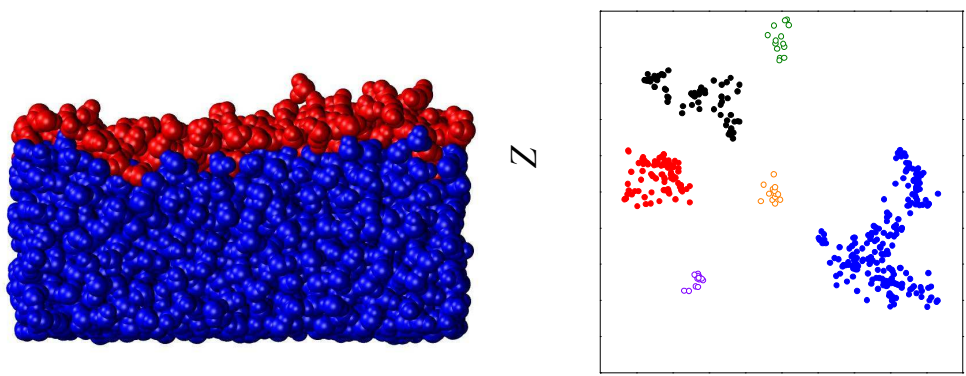

Y 